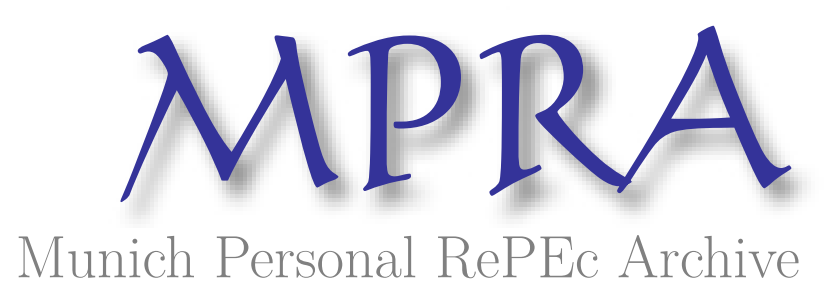

\title{
Winner-take-all, take-most or pay-some? \\ Optimal protection of innovation in a dynamic model of product development
}

Billette de Villemeur, Etienne and Ruble, Richard and Versaevel, Bruno

September 2017

Online at https://mpra.ub.uni-muenchen.de/81745/

MPRA Paper No. 81745, posted 02 Oct 2017 21:04 UTC 


\title{
Winner-take-all, take-most or pay-some? Optimal protection of innovation in a dynamic model of product development
}

\author{
Etienne Billette de Villemeur, Richard Ruble and Bruno Versaevel*
}

September 13, 2017

\begin{abstract}
In a model of investment in product development in duopoly we study the implications of different costs of innovating and imitating for firm strategies and optimal IP protection, relating these to the dynamic characteristics of a stochastic demand. A critical relative cost is identified that determines whether strategic competition takes the form of attrition or preemption, with industry value being maximized when firms neither stall nor hasten entry. Provided that demand growth and volatility are sufficiently low, as typically arises in mature industries, it is socially desirable to provide innovators with complete protection (winner-take-all), implying a preemption race. But when demand is rapidly expanding and highly unpredictable a social optimum can involve a low level of protection, implying attrition, albeit with a positive lower bound for the optimal level of imitation cost (winner-pays-some). Industry profits increase if firms can commit not to seek stronger IP protection once they have innovated, providing a rationale for open standards. While buyouts have ambiguous welfare effects, simple licensing schemes are welfare improving.
\end{abstract}

JEL classification: G31, L13, O33

Keywords: dynamic oligopoly, patent policy, preemption, real options

${ }^{*}$ Billette de Villemeur: LEM, Université de Lille, F-59655, France. Ruble and Versaevel: EMLYON Business School, F-69134 (primary affiliation) and CNRS, GATE Lyon Saint-Etienne, Ecully F-69130, France. Corresponding author: ruble@em-lyon.com. We are grateful to Xavier Boutin, Yann Braouezec, Benoît Chevalier-Roignant, David Encaoua, Thilo Meyer-Brandis, Dean Paxson, Régis Renault, Jacco Thijssen, Mihkel Tombak, and Lenos Trigeorgis as well as seminar participants at Paris-Dauphine university, IESEG business school, LMU and TU München, and participants at the 2016 AFSE meeting and Northwestern Annual Conference on Innovation Economics, 2015 International Conference on Real Options and 2014 EARIE meeting for valuable suggestions. 


\section{Introduction}

When developing an invention into a commercial product requires significant resources, only a few firms may jockey for positions in an industry either as a first-mover or as a second entrant. In these circumstances the timing of product introductions is determined by investment strategies that are driven by the relative costs of innovation and imitation and respond to such policy variables as the strength of intellectual property (IP) rights, whose effectiveness must hinge therefore on dynamic characteristics of the final product market.

In a model of innovation focusing on competing investments in the development of a new product, we study the implications of differences in the costs of innovating and imitating for the strategies of firms when their roles as an innovator or imitator are endogenous, in order to characterize the regulator's choice of optimal IP protection level. By identifying the role played by the drift and the volatility of demand in this context we are able to shed light on the timing of innovative and imitative investment and its implications for welfare, so as to contribute a novel perspective on the necessity of strong IP protection in certain types of markets.

A high degree of IP protection that induces a preemption race between firms is seen to constitute a second-best from a welfare standpoint under general conditions that are satisfied in common oligopoly models if demand growth and volatility are sufficiently low, as typically occurs in mature industries. When the level of these demand characteristics is sufficiently low in fact, it is socially desirable to provide innovators with complete protection and let strategic investment take the form of a winner-take-all contest. Conversely, attrition arises if innovation has positive spillovers for the imitating firm, and it is all the more likely when demand growth and volatility are high, a case that corresponds to the circumstances most commonly associated with innovative industries. Even then an optimal level of IP protection still sets a positive cost of imitating that we identify, so that dynamic competition between firms should always be winner-pay-some in nature.

Specifically, we investigate the exercise of strategic growth options by two initially identical firms contemplating the development of a product for a new market in which they are potential horizontal competitors. ${ }^{1}$ Investment levels are fixed and irreversible and product development occurs in a context of uncertainty, as market demand evolves stochastically over time through a scale parameter that follows a geometric Brownian motion. ${ }^{2}$ Both firms independently choose invest-

\footnotetext{
${ }^{1}$ Our focus on industries in which firms are horizontal competitors is therefore complementary to research on cumulative innovation such as Green and Scotchmer [15].

${ }^{2}$ We thus focus on the effect market uncertainty rather than R\&D uncertainty on innovation incentives, the latter having been extensively studied in the patent race literature (see e.g. Denicolò [6]).
} 
ment thresholds that determine the timing of their investment, and once performed investments result in a perpetual profit flow whose magnitude at any moment depends on the number of active firms. We thus study a real option game ${ }^{3}$ but depart from existing work by introducing an ex-post asymmetry through the differing fixed costs of innovation and imitation that firms face. We do not restrict the relative magnitude of these fixed costs, so the model allows for either a broad level of IP protection which implies a relatively high cost of imitation, or significant spillovers that result in a comparatively low cost of imitation.

Through the difference between the costs of innovating and imitating we parametrize first- and second-mover advantage in a parsimonious way, which allows us to nest in the same framework two classic games of timing (the deterministic models of attrition and preemption of Hendricks et al. [17] and Fudenberg and Tirole [11]) whose combination and formulation in the stochastic case present several challenges (Thijssen et al. [34], Riedel and Steg [29]). In order to allow a tractable welfare analysis of the effect of the level of IP protection on firm investment incentives and industry outcomes, we consider a timing game in which the strategies of firms consist of investment thresholds or hurdle rates that determine stochastic investment times, for which we provide a simple strategic form and characterize the unique symmetric equilibrium in the investment threshold choices of firms (in Section A.5 we complement the strategic form with a more technical discussion of extended mixed strategies and a dynamic view of the investment game).

Our first result relates equilibrium strategic investment to the relative costs of innovation and imitation (Proposition 1), and we identify a critical level of imitation cost that determines whether strategic competition between firms takes the form of attrition or preemption. For sufficiently low or high values of the cost of imitation, we find that strategic competition between firms has the form of a standard attrition or preemption game. A sufficiently low imitation cost leads to a situation of attrition as firms seek to enter second, delaying product introduction and inducing more or less rapid imitation. ${ }^{4}$ Conversely a sufficiently high imitation cost leads to a situation of preemption as firms seek to enter first, accelerating product introduction but generally delaying imitation. For intermediate imitation cost values, strategic competition takes the form either of a waiting game in which firm investment thresholds are continuously distributed over a disconnected support, or of a preemption race in which an attrition phase may occur off the equilibrium path.

\footnotetext{
${ }^{3}$ Chevalier-Roignant and Trigeorgis [4] provide a thorough presentation of such games, in which firms balance the value of retaining flexibility in the face of uncertainty with the strategic incentive to invest early.

${ }^{4}$ Our model thus encompasses dynamics akin to those described by Scherer (1980, quoted in Fudenberg and Tirole [11]) as "each industry member holding back initiating its R\&D effort in the fear that rapid imitation by others will be encouraged, more than wiping out its innovative profits."
} 
Attrition occurs provided that innovation has positive spillovers, if product market competition is sufficiently weak, and market growth and volatility are sufficiently high. This is because high growth and volatility raise the option value of delaying investment, eventually compensating for the lost monopoly profit phase if the firm manages to enter second and imitate instead. A useful additional result concerns the optimal balance between first- and second-mover advantage from the standpoint of the industry. Under both attrition and preemption, positional rents are dissipated in equilibrium, so that expected industry value is maximized if the imitation cost attains a critical level at which there is neither a race to preempt nor a war of attrition, as firms thus do not compete for positional rents by unduly either rushing or waiting to innovate (Proposition 2).

Because the model has a tractable equilibrium solution we are able to study the choice of socially optimal IP protection levels by a regulator who adjusts the cost incurred when imitating an innovation. The welfare trade-offs associated with raising imitation cost are largely intuitive. Aside from the effect on industry profit described above, a higher imitation cost makes the investment game more preemptive, accelerating innovation but also increasing the lag before imitation occurs with corresponding implications for consumer surplus. Viewed as a function of the imitation cost chosen by the regulator, social welfare generally has local maxima in both the attrition and preemption ranges, and either type of stationary point may constitute a global maximum (Proposition 3). We are able to identify a lower bound on the socially optimal imitation cost as well as to characterize the optimal imitation cost under preemption. We further identify conditions on economic primitives for one form or another of dynamic competition to be optimal: social welfare maximization involves attrition if the lack of competition is very damaging for consumers (like when the monopoly operator practices perfect price discrimination), and preemption is optimal when the introduction of additional competition does not bring much to consumers (for example, when there is product market collusion under duopoly).

It is challenging to draw more general conclusions regarding optimal IP levels, but we are able to show in our model that, provided that the static entry incentive is socially excessive - as occurs in the presence of a business-stealing effect - preemption is optimal when market growth and volatility are sufficiently low (Proposition 4). In a model of irreversible investment these market characteristics are associated with a greater discounting parameter so this result seems intuitive at first glance, since the adverse effect of higher imitation cost on welfare primarily stems from the ensuing delay in imitation which clearly decreases with discounting. However the welfare realized under attrition does not have a closed-form expression, and it is only by identifying an upper bound on this welfare expression that we are able to establish our result. Moreover, when the optimal imitation cost level induces preemption, the closed form expression for the (second-best) optimal 
imitation cost that we obtain leads us to ascertain when the optimal form of competition for an industry is to set an arbitrarily high (winner-take-all) cost of imitation. Our model thus provides an argument for strong IP rights in certain industries, which is grounded in specific demand characteristics (low growth and uncertainty). In addition, we are able to identify a positive lower bound for the optimal level of imitation cost, implying that free imitation is always socially costly (winner-pay-some).

Finally, we discuss several extensions of the investment game by incorporating a broader set of firm decisions. First we endogenize the cost of imitation by allowing the innovator to pursue patent protection more aggressively or to make reverse engineering of its product more difficult, and find that a higher baseline cost of imitation reduces the effort exerted by innovators to raise entry barriers. ${ }^{5}$ In addition, firms are shown to gain from coordinating ex-ante not to introduce subsequent complexity, a policy we refer to as an open standard (Proposition 5). We also discuss contracting between innovator and imitator that can take the form either of a buyout or of a license agreement, and show that efficiency always increases in the latter case (Proposition 6).

Our paper is related to early research on innovation incentives, which addresses the issue of optimal IP protection. Among the influential articles in this literature, Klemperer [23] and Gilbert and Shapiro [13] study the socially optimal trade-off between patent length and breadth for a given discounted present value to the innovator, and Gallini [12] introduces a cost of imitation that the regulator may control as a third patent instrument. We similarly emphasize the role of the cost of inventing around and determine an optimal level of patent breadth, but in contrast with these earlier approaches we allow for the possibility that firms in imperfectly competitive industries may wait to invest rather than developing a new product right when its net present value is positive, and derive the consequences of optimal investment timing for welfare. Closer to our work is Denicolò [6]'s model of optimal IP protection in a patent race, as we also formalize innovation and imitation as the outcome of a non-cooperative interaction that precedes market competition, though in contrast to his model we allow for second-mover advantage and possible attrition, which we show is likely to arise in industries with high growth and volatility.

Our analysis relates to several other papers that develop a welfare comparison across two alternative policy regimes, namely a strict winner-take-all regime where only the first firm to innovate receives a patent, and a more permissive winner-take-most regime where late investors are allowed to compete with the first before its patent expires. In La Manna, Macleod, and de Meza [24] firms spend a fixed initial amount in R\&D that determines a probability of inventing

\footnotetext{
${ }^{5}$ This extension also has implications for a patent system that allows firms to self-select protection levels, as proposed by Encaoua et al. [9], so as to endogenously raise the investment cost of follower firms.
} 
at a future date. Simple cost and demand conditions, such as constant returns to scale and a linear demand, are identified for the permissive regime to be welfare superior. Henry [18] introduces a mechanism whereby a late inventor can share the patent with the innovator within a given time period. When adjusted, together with other policy instruments, this mechanism is socially beneficial under mild conditions, notably with a linear demand and quantity competition. However, in a model where firms incur a flow cost, in Denicolò and Franzoni [7] it is the strict patent regime that is found to be optimal in broad set of circumstances, in particular when demand is linear, product market competition is weak, and duplication flow costs are large. Our approach is consistent with these contributions, although we do not compare discrete policy regimes and rather seek to characterize the optimal degree of IP protection in a continuous measure of the relative imitation cost, with the winner-take-all regime occurring as a limit case. Moreover, the model of investment under uncertainty allows us to connect optimal protection with measurable demand characteristics that were not considered in this stream of literature. Accordingly, our results depart from the comparison of contrasted policy regimes and point rather to adapting IP protection to measurable properties of the dynamics of markets.

Our paper is also close to studies of the effect on investment decisions of informational spillovers which can imply a second-mover advantage. Katz and Shapiro [22] is an early model in which a firm benefits from its rival's innovative activity through post-development dissemination of knowledge in a deterministic framework. Hoppe [21] allows for uncertainty regarding the success of new technology adoption whereas in Thijssen et al. [33] information regarding the value of a project arrives continuously over time, and the second investor faces an identical cost but learns about a project's true profitability from the first. Femminis and Martini [10] allow for a disclosure lag of random duration before the follower receives the information. In all of these models both preemption and attrition can occur as in ours, depending on the level of spillovers, but aside from Thijssen et al., all focus on pure strategy equilibria, whereas we characterize the symmetric mixed strategy equilibrium - a reasonable methodological choice when firms are assumed to be ex-ante symmetric, which provides what seems to us to be a more intuitive characterization of socially optimal IP protection.

Finally, similar licensing and reverse engineering decisions to those that we examine in our final extensions are found in Mukherjee and Pennings [27] and Henry and Ruiz-Aliseda [19], albeit once the roles of firms as incumbents or potential entrants have been determined, so that unlike in our approach their focus is on inherently asymmetric firms.

In Section 2 we describe the pharmaceutical sector as a natural example to examine in light of our analysis. Section 3 presents our model and characterizes the symmetric equilibrium. Section 
4 studies welfare when a regulator uses an instrument such as breadth of IP protection to regulate the cost of imitation. In Section 5 two extensions are discussed. Section 6 concludes.

\section{An example: contrasted market conditions in the pharmaceuti- cal industry}

The theoretical model that we develop points towards a policy prescription that consists in tailoring IP protection to general industry or market segment characteristics. The biopharmaceutical sector, in which $\mathrm{R} \& \mathrm{D}$ spending and innovation play a critical role, provides a natural industry example to examine in light of our analysis, as significant steps have already been taken in order to adjust IP protection in response to identifiable categories of market conditions. For instance for orphan drugs and rare disease development, the U.S. Food and Drug Administration has enacted an enhanced form of IP protection (Orphan Drug Exclusivity) together with a tax credit that lowers the costs of clinical trials (Grabowski et al. [14]). Our analysis offers theoretical support to regulatory measures of this kind that adapt the relative cost of innovation and imitation to a market's specific characteristics, and which could also involve patent narrowing in case of high demand growth and volatility.

Pharmaceutical firms face market conditions that impact product introductions and that can vary significantly across geographic areas. In low- and middle-income countries, economic and demographic drivers often imply high demand growth, but political instability can also result in less demand predictability than in high-income economies, and thus discourage the industry from introducing new treatments or preventives. Managers of big pharmaceutical companies are very aware of such market characteristics, and emphasize that although "pharmaceutical markets in key emerging economies, such as China, India, and Brazil, are expanding at rates of more than 12 percent per year (...) uncertain demand, and political and economic instability in some countries have deterred private investors for decades" (Witty [36], pp. 118 and 124).

In such circumstances, initiatives have been taken that alter the relative cost of imitation in order to encourage generic competition by local producers. For example, in order to increase access to antiretroviral drugs to treat HIV infection in the developing world, over the last decades political mobilization has facilitated the production of generic versions of the medicines patented in developed countries (Hoen et al. [20]). However, any adjustment in the IP protection regime also modifies innovation incentives, and absent a theoretical reflection as conducted in the present paper it is not clear how such changes should relate to demand characteristics in order for firms' 
decisions to result in greater social value. Our results show that when demand is rapidly expanding and highly uncertain, although shifting to weaker IP protection may reduce expected industry value (Corollary 2 and Proposition 2) it has the potential to improve social welfare, provided that patents are not abolished altogether and thus imitation costs are not too low (Proposition 3), and that it is in more mature markets such as those of high-income economies that strong IP protection should be upheld.

Within this same industry, conditions of imitation for drugs strongly differ from those for vaccines. ${ }^{6}$ Pharmaceutical firms rely on intellectual property rights in order to increase the costs of imitators for new drugs "which otherwise could be copied more easily than products whose production processes can be kept secret, or for which the time and relative expense needed to copy the invention are much higher" (Scherer and Watal [31], p. 4). If such patent protection is not available, a generic product can be introduced at a much lower fixed cost than incurred by the branded product supplier. However, this ease of imitation is not found in the case of vaccines, which are made from living micro-organisms, and unlike drugs "are not easily reverse-engineered, as the greatest challenges often lie in details of production processes that cannot be inferred from the final product," implying that "there is technically no such thing as a generic vaccine" (Wilson [35], p. 13).

The regulatory implication is that the imitating firm must reinvest in clinical trials which can involve subjects in the tens of thousands, and in complex manufacturing facilities that comply with demanding regulatory standards, before applying for approval of the product by the regulator. The fixed cost that must be incurred by a new entrant for the delivery of a follow-on vaccine can thus be prohibitively high, which is consistent with less systematic reliance on patenting by established firms than in the drugs segment. We return to this issue in light of the theoretical model in Section 5.1 , where the imitation cost is assumed to depend on the innovator's choice of protection level.

\section{A model of the timing of new product development in duopoly}

This section describes our model of strategic investment in product development that reflects the characteristic features of innovation and imitation identified in the introduction and illustrated in the industry example above. Assumptions regarding industry structure are presented in Section

\footnotetext{
${ }^{6}$ Another salient characteristic of the pharmaceutical industry is the R\&D uncertainty that is introduced by late-stage clinical trials regarding the outcome of a research project, most often after significant costs have already been sunk, but the focus of our model is on market uncertainty which is also important (see footnote 2).
} 
3.1, those regarding firm conduct and the investment game are presented in Section 3.2, and equilibrium is characterized in Section 3.3.

\subsection{Industry structure}

Two identical firms seek to enter a market by introducing their version of a novel product. Organizational constraints preclude a firm from selling two variants of the product and technological or regulatory barriers shield both firms from further entry. Development of the new product involves an irreversible investment in the face of uncertainty regarding future demand levels.

The introduction of the product generates a baseline profit flow $\pi_{M}$ when a single firm $i, i \in$ $\{1,2\}$ is active and $\pi_{D}$ when both are, with $0<\pi_{D}<\pi_{M}$. Flow profit at time $t$ takes the form $\pi_{M} Y_{t}$ or $\pi_{D} Y_{t}$ where the multiplicative component $Y_{t}$ is a measure of market size and is assumed to follow a geometric Brownian motion, $d Y_{t}=\alpha Y_{t} d t+\sigma Y_{t} d Z_{t}$ where $\left(Z_{t}\right)_{t \geq 0}$ is a standard Wiener process, $\alpha$ is the drift of the process and the volatility parameter $\sigma>0$ reflects the notion that demand for a new product evolves in a context of uncertainty. Profit flows instantaneously and with certainty once investment has occurred. Firms have a common and constant discount rate assumed to be large enough that the investment problem is economically meaningful $(r>\alpha)$.

Introducing the new product involves a positive irrecuperable fixed cost $I$ for the the first firm that invests to serve demand, i.e. for the innovator. If its rival has innovated, a firm can invest afterwards (even immediately) as a second entrant, i.e. as an imitator, and introducing the imitative product also involves a fixed cost $K$ which is also irrecuperable. The imitator's fixed cost is allowed to be either higher or lower than the innovator's, and we allow for the extreme cases of costless or arbitrarily costly imitation $(K=0$ or $K=\infty)$. The cost of imitation typically includes various standard setup costs associated with bringing a product to market such as dedicated plant and equipment, marketing expenditures, and so forth, as well as the cost of developing the firm's version of the new product. If the second firm can develop the same product independently, imitation should be no more expensive than innovation in the absence of IP, and even cost strictly less to the extent that there are knowledge spillovers $(K \leq I)$. When IP protection is sufficiently strong however, imitators must invent around any patents held by the innovator and the second mover can incur higher entry costs than the leader $(K>I) .^{7}$

\footnotetext{
${ }^{7}$ Other circumstances can lead to asymmetric fixed costs for initially identical firms. For example, if developing the new product involves an input in scarce supply, the imitator typically face a higher cost. Alternatively, Billette de Villemeur et al. [2] show that if the cost of investment is determined endogenously by a monopoly input supplier, the input price is endogenously discounted for the first firm that invests.
} 


\subsection{Firm strategies and payoffs}

Firms play a game of timing whose normal form is studied in this section for simplicity of exposition. This framework provides enough structure to derive the welfare results in Section 4 and adequately reflects economic behavior so long as players do not make empty threats. We verify that this is indeed the case by developing a dynamic view of the same game in which players use extended mixed strategies as per Fudenberg and Tirole [11] in the appendix. This dynamic view does not pose any novel difficulties provided that the firms invest at the first-hitting times of the stochastic process, but it is somewhat lengthy to develop and interested readers may therefore refer to Section A.5.

Each firm chooses an investment threshold that triggers its investment when it is reached for the first time and in the absence of rival investment, but which it is free to revise if rival investment occurs. Each firm's investment behavior is therefore conditioned on the dynamic evolution of the market but open-loop with respect to any rival move until an investment occurs. ${ }^{8}$ Once an investment occurs, any remaining firm may either respond by investing immediately or revise its strategy in the continuation phase which is a single-firm decision problem. Industry dynamics thus typically consist of a period of inaction before either firm has entered and over which the main strategic interaction between the firms unfolds, followed by a possible monopoly phase and finally a duopoly phase once both firms have entered.

It is assumed that $Y_{0} \leq(r-\alpha) I / \pi_{M}$, so that the demand level when the game begins is sufficiently small for firms to prefer delaying investment. The strategy of firm $i, i=1,2$, consists of an entry threshold $Y_{i} \in\left[Y_{0}, \infty\right)$ that triggers investment when it is reached for the first time, absent prior rival entry. Investment thresholds determine the roles of firms as innovator or imitator and the (stochastic) moment at which the product is initially introduced, and once rival investment (innovation) has occurred any remaining firm is able to revise its investment threshold in a continuation (imitation) phase. The strategies $Y_{i}$ determine a stochastic time at which the firm plans to invest, which is a first hitting time $\tau\left(Y_{i}\right):=\inf \left\{t \geq 0 \mid Y_{t} \geq Y_{i}\right\}$.

If investment by one of the firms occurs at a value of the scale parameter $Y_{t}=y$, the optimal policy of any firm that has not yet entered is to invest when the market size has reached an optimal threshold, denoted $Y_{F}$. Standard arguments (see Section A.1) establish that the continuation payoff of such a (follower) firm at a given time $t$ is

$$
C(y)= \begin{cases}A_{C} y^{\beta} & , \quad y<Y_{F} \\ \frac{\pi_{D}}{r-\alpha} y-K & , \quad y \geq Y_{F}\end{cases}
$$

\footnotetext{
${ }^{8}$ This difference with Reinganum [28]'s notion of open-loop strategies plays a key role in the investment game.
} 
where $\beta$ is shorthand for the function of parameters

$$
\beta(\alpha, \sigma, r):=\frac{1}{2}-\frac{\alpha}{\sigma^{2}}+\sqrt{\left(\frac{1}{2}-\frac{\alpha}{\sigma^{2}}\right)^{2}+\frac{2 r}{\sigma^{2}}},
$$

$Y_{F}:=(\beta(r-\alpha) K) /\left((\beta-1) \pi_{D}\right)$ is the optimal investment threshold of a firm that earns a duopoly profit stream upon entry and $A_{C}:=\beta^{\beta} \pi_{D}^{\beta} /(\beta-1)^{\beta-1}(r-\alpha)^{\beta} K^{\beta-1}$. The function in (2) is a standard expression in models of investment under uncertainty and satisfies $\beta>1$ and $\partial \beta / \partial \alpha, \partial \beta / \partial \sigma<0 .^{9}$

Given the starting value of the demand parameter, if initial investment occurs at a threshold $Y \geq y$ and any investment in the continuation phase occurs at the threshold $\max \left\{Y, Y_{F}\right\}$, the expected payoff of a leader (or innovator) at time $t=0$ is

$$
\begin{aligned}
L(Y) & =\mathbb{E}_{y}\left(\int_{\tau(Y)}^{\tau\left(\max \left\{Y, Y_{F}\right\}\right)} \pi_{M} Y_{s} e^{-r s} d s-e^{-r \tau(Y)} I+\int_{\tau\left(\max \left\{Y, Y_{F}\right\}\right)}^{\infty} \pi_{D} Y_{s} e^{-r s} d s\right) \\
& =\left(\frac{\pi_{M}}{r-\alpha} Y-I\right)\left(\frac{y}{Y}\right)^{\beta}-\frac{\pi_{M}-\pi_{D}}{r-\alpha} \frac{y^{\beta}}{\left[\max \left\{Y, Y_{F}\right\}\right]^{\beta-1}}
\end{aligned}
$$

(see Section A.2 for derivations). The first summand in (3) corresponds to the discounted value of perpetual monopoly profits for a firm that invests at the threshold $Y$ and the second is a correction term corresponding to the reduction in profit flow stemming from the rival's anticipated entry. Given that innovation occurs at the threshold $Y$, the payoff to any remaining firm from becoming a follower at the moment that $Y$ is first reached is

$$
F(Y)=C\left(\max \left\{Y, Y_{F}\right\}\right)\left(\frac{y}{\max \left\{Y, Y_{F}\right\}}\right)^{\beta} .
$$

If both firms invest at the same moment the cost of investment is assumed to be $I$ for each. This may reflect either a legal tolerance for independent discovery, or a lag for information spillovers to occur that we do not model explicitly. ${ }^{10}$ The initial expected payoff to firms is then

$$
\begin{aligned}
M(Y) & =\mathbb{E}_{y}\left(\int_{\tau(Y)}^{\infty} \pi_{D} Y_{s} e^{-r s} d s\right) \\
& =\left(\frac{\pi_{D}}{r-\alpha} Y-I\right)\left(\frac{y}{Y}\right)^{\beta} .
\end{aligned}
$$

\footnotetext{
${ }^{9} \mathrm{~A}$ standard result regarding $\beta$ is that for any $Y \geq y$, the expected discounted value of a monetary unit received when the process first hits the threshold $Y$ is $\mathbb{E}_{y} e^{-r \tau(Y)}=(y / Y)$. As volatility decreases this discounted value converges to the standard deterministic continuous time discount term, i.e. $\lim _{\sigma \rightarrow 0}(y / Y)^{\beta}=e^{-r \tau(Y)}$.

${ }^{10} \mathrm{An}$ alternative assumption would be that IP protection and spillovers are determined randomly when investments are simultaneous so the expected fixed cost at the moment of investment is $(I+K) / 2$. In this case the first intersection of $L$ and $M$ is then no longer at $Y_{F}$ rendering the algebraic expressions more complex, but the general configuration of payoffs and equilibrium upon which our results are based is similar.
} 


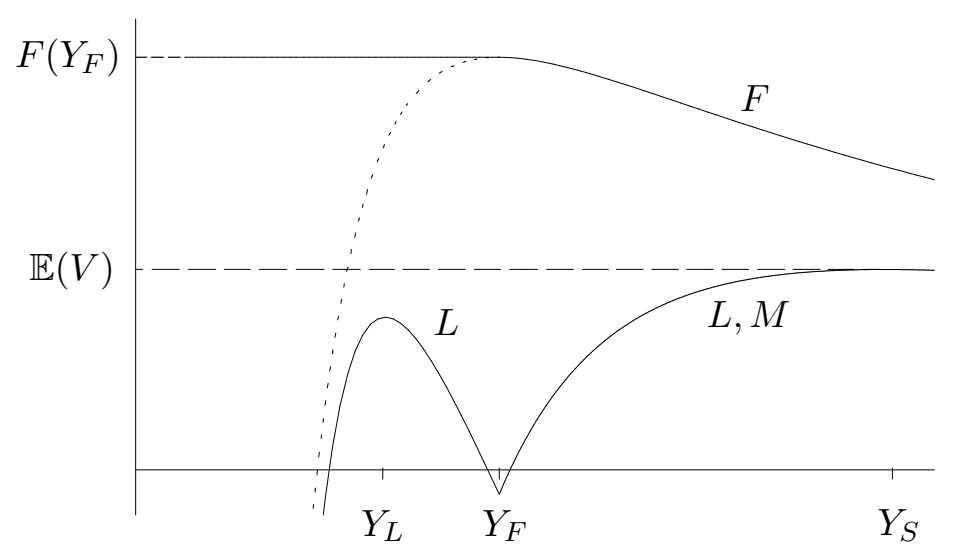

Figure 1: Attrition, $K \leq \widetilde{K}, Y_{S}$ is a global maximum of the leader payoff, innovation thresholds are distributed over $\left[Y_{S}, \infty\right)$, and imitation occurs immediately after.

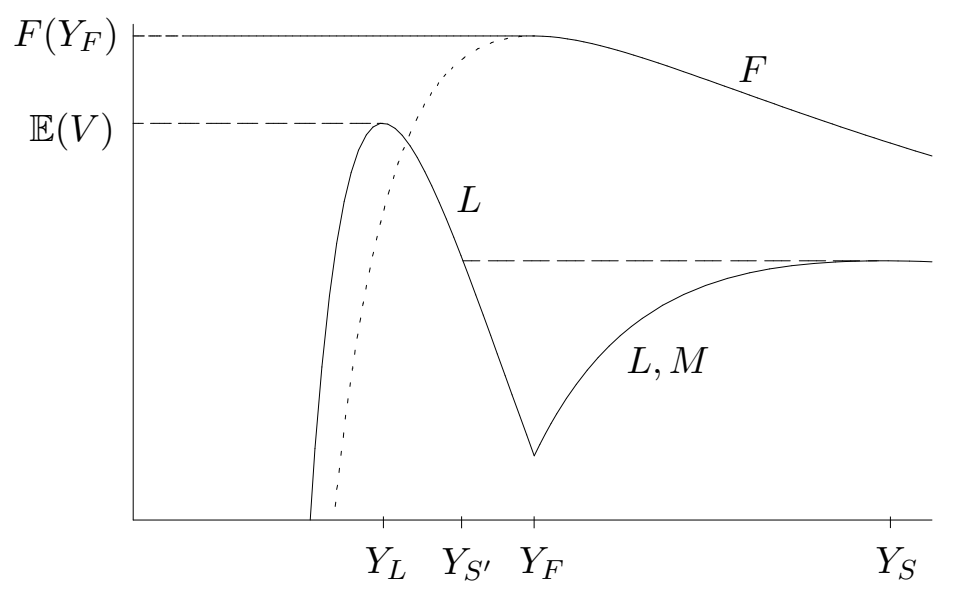

Figure 2: Attrition, $\widetilde{K}<K<\widehat{K}, Y_{S}$ is a global maximum of the leader payoff, innovation thresholds are distributed over $\left[Y_{L}, Y_{S^{\prime}}\right] \cup\left[Y_{S}, \infty\right)$, imitation occurs either at $Y_{F}$ if the innovation threshold is in $\left[Y_{L}, Y_{S^{\prime}}\right]$, or immediately otherwise. 


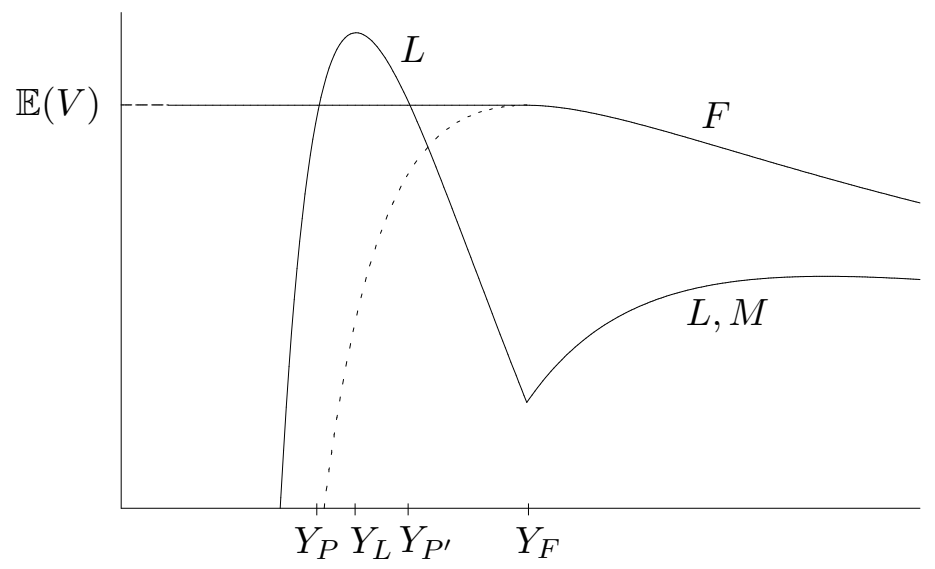

Figure 3: Preemption, $\widehat{K}<K<I$, innovation occurs at $Y_{P}$ and imitation at $Y_{F}$. There is war of attrition off the equilibrium path (over $\left.\left(Y_{P^{\prime}}, \infty\right)\right)$.

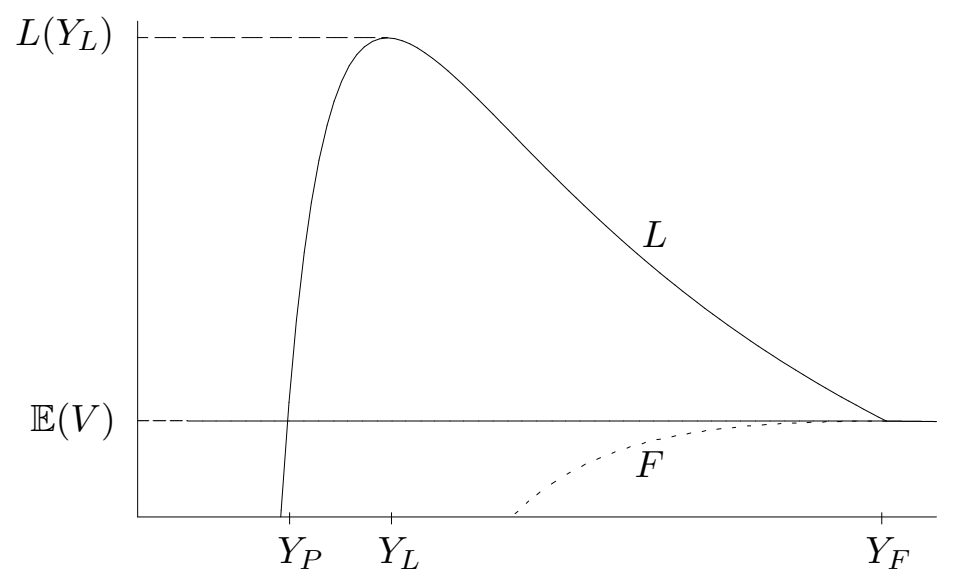

Figure 4: Preemption, $K \geq I$, innovation occurs at $Y_{P}$ and imitation at $Y_{F}$. The dotted curve represents $F(Y)$ whereas the expected payoff of firms is equal to the concentrated follower payoff $F\left(Y_{F}\right)$ (here with $K=I$ ).

The behavior of $L, F$ and $M$ for different values of $K$ determines the nature of the investment 
game. There exist three critical levels of the imitation cost, $\widetilde{K}<\widehat{K}<I,{ }^{11}$ that determine four typical payoff configurations which are represented in Figures $1-4$.

For $K<\widetilde{K}$ (Figure 1), ${ }^{12} F>L$ for all $Y \geq y$ and $L$ has a global maximum at $Y_{S}:=$ $(\beta(r-\alpha) I) /\left((\beta-1) \pi_{D}\right)$. For $\widetilde{K}<K<\widehat{K}$ (Figure 2$), F>L$ for all $Y \geq y$ but $L$ has a global maximum at $Y_{L}:=(\beta(r-\alpha) I) /\left((\beta-1) \pi_{M}\right)$. Note also that $L$ is not monotonic to the right of its global maximum in this case. For $\widehat{K}<K<I$ (Figure 3), $F<L$ over an interval denoted $\left(Y_{P}, Y_{P^{\prime}}\right)$ that includes $Y_{L}$. Finally, for $K>I$ (Figure 4$), F<L$ for all $Y \geq y$.

Although the specific relationships between the payoffs are complex there is a broad intuition underlying these the figures. Beginning with an extremely low cost of imitation $(K=0)$, the payoff to following is globally higher than that of leading $(F>L)$. Raising the level of $K$ then increases the follower investment threshold $Y_{F}$ and shifts $F$ downward, but conversely this higher investment threshold lengthens the monopoly phase and raises the payoff to leading before follower investment occurs, shifting $L$ upward over the range $\left(y, Y_{F}\right)$. Ultimately if the cost of imitation becomes large enough $(K>I)$ it is the payoff to leading that is globally higher than that of following $(F<L)$.

A last element of structure must be introduced before the payoffs and the investment game can be fully described. It is well-known that in preemption games, firms face a coordination problem if both seek to invest at the same threshold when it would be optimal for only one to do so, i.e. if at some $Y_{1}=Y_{2}=Y, L(Y) \geq F(Y)$ and $F(Y)>M(Y)$. A standard solution proposed by Fudenberg and Tirole [11] is for players to use augmented mixed strategies that reflect the coordination that arises in a discrete-time game and which is not captured directly in a continuous time model. For simplicity we proceed somewhat differently and introduce a specific tie-breaking rule to determine investment outcomes in such cases, leaving the formulation of extended mixed strategies for the appendix. Following Thijssen [32], the tie-breaking rule is chosen so that payoffs are consistent with those that obtain with augmented mixed strategies by imposing rent equalization.

That is to say, if firms face such a coordination problem at the threshold $Y$ the probability that either firm invests first (as a leader), $p$, is chosen so as to solve $p L(Y)+p F(Y)+(1-2 p) M(Y)=$

\footnotetext{
${ }^{11}$ See Section A.2.3 for a derivation of the critical values $\widetilde{K}=\left(\beta\left(\left(\pi_{M} / \pi_{D}\right)-1\right) /\left(\left(\pi_{M} / \pi_{D}\right)^{\beta}-1\right)\right)^{1 /(\beta-1)} I$ and $\widehat{K}=\left(\left(1+\beta\left(\left(\pi_{M} / \pi_{D}\right)-1\right)\right) /\left(\pi_{M} / \pi_{D}\right)^{\beta}\right)^{1 /(\beta-1)} I$.

${ }^{12}$ Figure 1 is drawn assuming $K>\left(\pi_{D} / \pi_{M}\right) I$. Below this value, $Y_{F}<Y_{L}$ and $F$ is decreasing over $(y, \infty)$ but the key properties described in the text are unaltered.
} 
$F(Y)$ leaving firms indifferent between investing at that threshold and a subsequent follower role. This yields

$$
p(Y)=\left\{\begin{array}{lll}
\frac{F(Y)-M(Y)}{L(Y)+F(Y)-2 M(Y)} & \text { if } \quad L(Y) \geq F(Y) \\
0 & \text { if } \quad L(Y)<F(Y)
\end{array}\right.
$$

and accordingly $1-2 p\left(Y_{i} ; K\right)$ is the probability that a mistaken simultaneous investment occurs.

On the basis of these elements the initial payoff to firm $i$ from investing at the threshold $Y_{i}$ if the other firm invests at $Y_{j}$ is

$$
V\left(Y_{i}, Y_{j}\right)=\left\{\begin{array}{lll}
L\left(Y_{i}\right) & \text { if } & Y_{i}<Y_{j} \\
p\left(Y_{i}\right) L\left(Y_{i}\right)+p\left(Y_{i}\right) F\left(Y_{i}\right)+\left(1-2 p\left(Y_{i}\right)\right) M\left(Y_{i}\right) & \text { if } \quad Y_{i}=Y_{j} \\
F\left(Y_{j}\right) & \text { if } \quad Y_{i}>Y_{j}
\end{array}\right.
$$

and the investment game is represented by the normal form $(\{1,2\},[y, \infty) \times[y, \infty),(V, V))$.

\subsection{Equilibrium}

The investment game has a unique symmetric Nash equilibrium involving either pure or mixed strategies. The resulting representation of equilibrium investment behavior is therefore consistent with the seminal approaches of Fudenberg and Tirole [11] and Hendricks et al. [17] to preemption and attrition. In attrition games, authors have sometimes studied (pure strategy) asymmetric equilibria. ${ }^{13}$ This is appropriate, for example, if the same entry game is played in several independent markets and pre-play communication enables coordination between firms, but we do not allow here for such a possibility. As firms are taken to be initially symmetric, it also seems natural to suppose that initial investment behavior should be symmetric as well. Moreover the equilibrium that we characterize involves a smooth dependence of outcomes on imitation cost that is of compelling simplicity.

To get an intuitive sense of the nature of the investment game for different levels of the imitation cost, it is useful to refer again to Figures $1-4$. For low enough levels of $K$ (Figure $1, K \leq \widetilde{K}$ ), the leader payoff $L(Y)$ lies below the follower payoff $F(Y)$ for all $Y \geq y$ so firms prefer to follow, a situation of attrition. Investing first at any threshold $Y_{i}<Y_{S}$ is dominated by investing at $Y_{S}$, and from that threshold onward $L(Y)$ is decreasing as in the standard war of attrition. For moderately low levels of $K$ (Figure $2, \widetilde{K}<K<\widehat{K}$ ), the leader payoff is globally lower than the follower payoff so that firms also play a waiting game. Since $Y_{L}$ is a global maximum of $L(Y)$, investing at $Y_{S}$

\footnotetext{
${ }^{13}$ For instance Katz and Shapiro [22] and Hoppe [21] proceed this way.
} 
does not dominate lower thresholds, and in particular thresholds in $\left[Y_{L}, Y_{S^{\prime}}\right]$. Firms therefore engage in a nonstandard form of attrition, with investment thresholds continuously distributed over the range $\left[Y_{L}, Y_{S^{\prime}}\right] \cup\left[Y_{S}, \infty\right)$ where $L(Y)$ is decreasing. For high levels of the imitation cost (Figure 3, $\widehat{K}<K<I$ and Figure $4, I \leq K$ ), there exists a range of thresholds below $Y_{F}$ for which the leader payoff $L(Y)$ lies above the follower payoff $F(Y)$ for all $Y \geq y$ so firms prefer to lead, a situation of preemption. Initial investment then occurs at the preemption threshold $Y_{P}$, which is defined as the lowest threshold at which firms are indifferent between leading and following (it is the lower root of the condition $L(Y)=F(Y)$ ). If the imitation cost is moderately high (Figure 3) the preemption game is nonstandard in that firms engage in a waiting game off the equilibrium path if the threshold $Y_{P^{\prime}}$ is reached and no firm has yet invested, whereas with a higher imitation cost (Figure 4) is a standard preemption case in which the leader payoff never drops below the follower payoff to the left of $Y_{F}$.

The proposition below states these ideas formally and also separately identifies the pivotal case $K=\widehat{K}$ at which there is neither a first-mover advantage nor a second-mover advantage in the sense that $L\left(Y_{L}\right)=F\left(Y_{F}\right)$ ), which plays a central role in the welfare analysis of the next section.

Proposition 1 In the symmetric equilibrium of the duopoly investment game,

(i) if the cost of imitation is low $(K<\widehat{K})$ firms play a game of attrition. Equilibrium is in mixed strategies with innovation thresholds distributed continuously over $\left[Y_{S}, \infty\right)$ if $K \leq \widetilde{K}$ and over a disconnected support $\left[Y_{L}, Y_{S^{\prime}}\right] \cup\left[Y_{S}, \infty\right)$ if $\widetilde{K}<K<\widehat{K}$. In the former case imitation is immediate whereas in the latter it occurs with lag at the threshold $Y_{F}$ with positive probability;

(ii) if the cost of imitation is high $(K>\widehat{K})$ firms play a game of preemption. Equilibrium is in pure strategies at the innovation threshold $Y_{P}$, and imitation occurs at the threshold $Y_{F}$;

(iii) at the critical imitation cost $\widehat{K}$ firms neither wait nor race to enter. Equilibrium is in pure strategies at the innovation threshold $Y_{L}$, and imitation occurs at the threshold $Y_{F}$.

In the symmetric equilibrium positive rent dissipation occurs whenever the firms play a nondegenerate timing game (when $K \neq \widehat{K}$ ). In an attrition regime, the rents of imitation that accrue to a follower are dissipated in expectation because firms wait before investing, earning the relevant leader payoff $L\left(Y_{L}\right)$ or $L\left(Y_{S}\right)$ depending on the level of $K$. In a preemption regime, the rents to a leader from innovation are dissipated as firms race to enter early, earning $F\left(Y_{F}\right)$. The expected value of firms in the investment game therefore has a simple characterization. This is stated in the following corollary, where the only difficulty stems from the fact that either $Y_{S}$ or $Y_{L}$ may represent a globally maximum of $L$ depending on the value of $K$. 
Corollary 1 In a symmetric equilibrium the duopoly investment game the expected payoff of firms are $\min \left\{F\left(Y_{F}\right), \max \left\{L\left(Y_{L}\right), L\left(Y_{S}\right)\right\}\right\}$.

Because the nature of the interaction between firms is determined by the level of the mitation cost $K$ relative to the critical imitation cost $\widehat{K}$, is it useful to have some sensitivity results with respect to the intensity of competition in the product market $\left(\pi_{M} / \pi_{D}\right)$ and parameters of the demand process $(\alpha$ and $\sigma)$.

Corollary 2 The more intense product market competition is and the lower demand growth and volatility, the more likely it is that preemption occurs, and conversely for attrition

$$
\frac{\partial \widehat{K}}{\partial\left(\pi_{M} / \pi_{D}\right)}<0, \frac{\partial \widehat{K}}{\partial \alpha}, \frac{\partial \widehat{K}}{\partial \sigma}>0 .
$$

To provide intuition for some of the latter comparative statics recall that the process $Y_{t}$ evolves stochastically and that there is an option value for firms to wait before investing that is positively related to drift and volatility. Provided that there is an inherent advantage to imitation $(K<I)$, for some parameter values and in particular for large enough levels of volatility (such that $K<\widehat{K}$ ), this option value outweighs any preemptive motive to secure monopoly rents. A similar reasoning holds if the drift in demand is sufficiently high. That is to say, an attrition regime is more likely in industries with greater trend growth and demand volatility. This observation is particularly noteworthy because it provides a countervailing force to several mechanisms that are highlighted in the rest of the paper. As the next sections show, institutional conditions such as IP protection and firm choices regarding both technology and licensing generally serve to make market entry regimes more preemptive and attrition relatively more rare. One would therefore expect attrition to seldom occur except in those industries with a significant enough degree of demand growth or demand uncertainty.

\section{Imitation cost, industry profit, and welfare}

The preceding section highlighted the role of the fixed cost of imitation in determining the nature of strategic competition and the equilibrium pattern of entry in an industry, and ultimately consumer surplus and welfare levels. This imitation cost is driven by several factors including technological conditions and the level of IP protection. It thus varies from industry to industry and can be influenced ex-ante by regulators, typically through a choice of patent breadth. These 
considerations raise the question of determining what may be socially desirable levels of imitation cost. At first glance this question appears to involve a simple trade-off since a higher imitation cost is wasteful but hastens innovator entry. However different effects arise with regard to the timing of imitator entry in the preemption and attrition regimes that need to be examined more carefully.

This section therefore studies the effect of $K$ on equilibrium strategies and outcomes. In order to express the social welfare function more compactly we introduce some further notation. Let $Y_{I}=\min \left\{Y_{1}, Y_{2}\right\}$ denote the threshold at which innovation occurs, so that in equilibrium by Proposition $1 Y_{I}=\widetilde{Y}_{A}, Y_{L}$ or $Y_{P}$ depending on the level of $K$ and where the random threshold $\widetilde{Y}_{A}$ in the attrition regime is distributed according to the density $G_{\wedge}(Y ; K)=1-(1-G \text {. }(Y))^{2}$, $G$. $=G_{a}$ or $G_{b}$ being the relevant equilibrium distribution of innovation thresholds (these are derived in Section A.3).

\subsection{Industry effects}

A useful preliminary step before conducting the welfare analysis is to characterize the effect of $K$ on industry performance, which allows us to derive an intermediate result regarding industry value. We begin by relating imitation cost to first- and second-mover advantage, and industry profitability.

A first observation that emerges from the model is that lower imitation cost is a necessary, but not a sufficient condition for second mover advantage. Too see why this is, consider an industry in which innovating and imitating firms have identical fixed costs. There is an inherent firstmover advantage for the innovating firm that stems from the monopoly phase that it enjoys over the range of thresholds $\left(Y_{L}, Y_{F}\right)$, and the degree of first-mover advantage in such an industry is determined by the relative importance of parameters like the relative monopoly rent $\left(\pi_{M} / \pi_{D}\right)$. In order for a second-mover advantage to arise in an industry, it must be that the relative cost of imitation $(K / I)$ in the input market is sufficiently low to compensate a firm for forgoing the period of monopoly profit it would earn by innovating. Thus a lower costs for imitators, the most likely case to arise absent IP protection, does not by itself ensure that firms have a first-mover advantage or that they will find it desirable to pursue so-called imitation strategies.

Next there are a number of monotone relationships between imitation cost and the equilibrium investment thresholds and outcomes in the model. First, the higher is the imitation cost, the higher is the standalone threshold for the follower firm $\left(Y_{F}\right)$. Note though that actual follower entry may or may not occur at this threshold, since innovation can occur at a threshold beyond this 
standalone threshold in an attrition regime where the support of mixed strategies extends beyond $Y_{F}$. The effect of imitation cost on the innovation threshold is consistently negative throughout the range of imitation costs. As imitation cost increases, in the attrition regime the distribution of innovator entry thresholds is shifted leftward whereas in the preemption regime the preemption threshold decreases, so innovation occurs earlier in both cases. The effect of higher imitation cost on the imitation threshold is monotone under preemption since $\partial Y_{F} / \partial K>0$, but this is not the case under attrition. This is because in an attrition regime, a greater imitation cost delays imitation only if the innovation threshold realization is low enough that there is a positive lag before imitation occurs (if $\tilde{Y}_{A} \leq Y_{F}$ ). Otherwise the onset of imitation, immediately after innovation, is itself random and its distribution (to the right of $Y_{F}$ ) is shifted leftward by an increase in imitation cost. Despite this complex effect of imitation cost on the timing of imitation, the gap (and therefore the expected time lag) between innovation and imitation thresholds, $\max \left\{Y_{F}, \widetilde{Y}_{A}\right\}-\widetilde{Y}_{A}$ is weakly increasing in $K$. To summarize, an increase in imitation cost may properly be said to accelerate innovative investment and the arrival of imitative investment conditional upon innovation having occurred.

Lastly, there exists a simple relationship between imitation cost and equilibrium industry performance. Because in the different regimes of attrition and preemption, competition between firms to secure either second- or first-mover advantages results in the dissipation of any positional rents, equilibrium firm and industry values have a straightforward expression (Corollary 1). Moreover, $\max \left\{L\left(Y_{L}\right), L\left(Y_{S}\right)\right\}$ which constitutes the equilibrium firm value in an attrition regime is either independent of (if $K \leq \widetilde{K}$ ) or increasing in $K$ (if $\widetilde{K}<K<\widehat{K}$ ) whereas $F\left(Y_{F}\right)$ which constitutes the equilibrium firm value in a preemption regime is decreasing in $K$. Since industry value is nondecreasing and subsequently increasing in $K$ under attrition up until $\widehat{K}$ and decreasing in $K$ thereafter under preemption, it is therefore only when the level of the imitation cost is such that neither of these regimes occurs $(K=\widehat{K})$ that investment thresholds are set optimally from the standpoint of industry profit. All else equal then, it is in those industries in which imitation cost reaches the level at which firms do not have an incentive to seek a positional advantage of either sort that industry value is maximized.

Proposition 2 Expected industry value is a quasiconcave function of imitation cost which is initially constant (over $(0, \widetilde{K})$ ) with a unique maximum when imitation cost is such neither attrition nor preemption occur (at $K=\widehat{K}$ ).

Proposition 2 states that there exists an initial range over which expected firm value is independent of imitation cost before increasing thereafter. In economic terms, this means that starting 
from a sufficiently low level, product introduction is more timely and firms benefit ex-ante from raising the fixed cost of imitation enough to shield an innovator from instantaneous imitation with positive probability. Moreover, and despite the wasteful nature of fixed costs for the second firm ex-post (to the extent that these include duplicative expenditures), raising fixed costs for the

second firm is strictly beneficial for the industry (over $(\widetilde{K}, \widehat{K})$ ) when the endogenous timing of investments is accounted for. Aside from the straightforward description of industry value it provides, Proposition 2 is also instrumental in establishing our main welfare results in the remainder of this section, which we turn to next.

\subsection{Social welfare and optimal protection of innovation}

We take the view that regulators can influence the relative cost of imitation through a choice of IP protection level which we interpret as patent breadth. With this instrument and provided that the natural imitation cost is not so high as to constrain the regulator's choice (it seems doubtful that imitation would be subsidized), the imitation cost may be viewed as a decision variable. We consider a second-best welfare benchmark in this section, in which firms are free to select their entry thresholds and product market output or prices so welfare is a function of the imitation cost, $K$.

To provide intuition for the welfare analysis, it is useful to decompose the expected welfare in the equilibrium of the investment game into three parts: expected industry value, the consumer surplus resulting from innovator entry and the consumer surplus resulting from imitator entry. By Proposition 2 the first of these components is single-peaked with a maximum at the critical imitation cost $\widehat{K}$. The two consumer surplus terms depend on imitation cost in roughly opposite ways, at least under preemption. A higher imitation cost unambiguously accelerates innovator entry which raises the consumer surplus from innovation term, so the second welfare component is monotonically increasing in $K$. Under preemption, higher imitation cost unambiguously delays imitator entry and the third welfare component is monotonically decreasing in $K$. But under attrition, the impact of imitation cost on imitator entry is more complex. Over that part of the support of mixed strategies where innovation occurs early enough $\left(\tilde{Y}_{A} \leq Y_{F}\right)$ an increase of imitation cost delays imitation and reduces the consumer surplus from imitation, but where innovation occurs late enough $\left(\widetilde{Y}_{A}>Y_{F}\right.$, which holds for the entire support of mixed strategies if $K<\widetilde{K}$ ), imitation is immediate and an increase of imitation cost raises the second consumer surplus term.

To study social welfare precisely, suppose that consumer surplus is scaled by the market size 
parameter $Y_{t}$ as is the case for firm profits. Let $C S_{M}$ and $C S_{D}$ then denote the unitary flows of consumer surplus under monopoly and under duopoly respectively. The social discount rate is assumed to be $r$ for simplicity, identical to that of firms. Recalling the innovation threshold $Y_{I}$ has a nondegenerate distribution for $K<\widehat{K}$, expected social welfare then has the specific form

$$
\begin{aligned}
& W(K)=\underbrace{2 \min \left\{F\left(Y_{F}\right), \max \left\{L\left(Y_{L}\right), L\left(Y_{S}\right)\right\}\right\}}_{\text {industry value }} \\
& +\underbrace{\mathbb{E} \frac{\mathrm{CS}_{M}}{r-\alpha}\left[\tilde{Y}_{I}\right]^{-(\beta-1)} y^{\beta}}_{\text {consumer surplus from innovation }}+\underbrace{\mathbb{E} \frac{\mathrm{CS}_{D}-\mathrm{CS}_{M}}{r-\alpha}\left[\max \left\{\tilde{Y}_{I}, Y_{F}\right\}\right]^{-(\beta-1)} y^{\beta}}_{\text {consumer surplus from imitation }} .
\end{aligned}
$$

The first summand in (8) is expected industry value. By Proposition 2 it is constant for $K<\widetilde{K}$ and strictly quasiconcave in $K$ thereafter with a maximum at $\widehat{K}$. The second term is the expected consumer surplus that results from innovative investment. As discussed above this term increases with $K$, since a higher imitation cost shifts the distribution of innovator entry thresholds (which is degenerate at $Y_{P}$ under preemption) leftward. The third term is the expected consumer surplus that results from the imitator's entry into the market, which involves a more complex dependence on $K$ as described above. Although there is no closed-form expression for the entire function (8), there is a semi-closed form for $K \geq \widehat{K}$ and we are able to bound the function over the remainder of its domain sufficiently in order to obtain a partial characterization of the social optimum (see Section A.6 for the full proof).

First, in the preemption range (for $K>\widehat{K}$ ) the innovator and imitator entry thresholds are respectively $Y_{P}$ and $Y_{F}$, the former of which does not have a closed-form expression. Nevertheless the semi-explicit form of $W(K)$ allows us to evaluate the unique local optimum of (8) under preemption, which we denote $K_{P}$. For a range of parameter values $\beta \in\left[\beta_{0}, \infty\right), \beta_{0}>1$, this optimum is a corner solution $\left(K_{P}=\infty\right)$. The social planner's imitation cost instrument is of too limited a reach to attain its welfare objective in such cases, so that for sufficiently large $\beta$ the socially optimal form of preemption is a winner-take-all contest. Since the greatest amount of preemption that the social planner can generate does not foster enough competition to induce firms to enter sufficiently early in such cases, a single firm is active ex-post whose investment threshold is determined by the threat of potential entry. On the other hand, if the discounting parameter is not too large $\left(\beta \in\left(1, \beta_{0}\right)\right)$ as occurs for instance if volatility is sufficiently high, $K_{P}$ is finite and strictly greater than $\widehat{K}$ so long as consumer surplus under monopoly is positive $\left(C S_{M}>0\right)$.

Second, even without an explicit form for $W(K)$ within this range, another possible maximum of welfare can be shown to arise under attrition. To establish this, because $W(K)$ is continuous it 
suffices to show that social welfare is decreasing to the left of the critical value $\widehat{K}$, which involves showing that $\lim _{K \rightarrow \widehat{K}^{-}} W^{\prime}(K)<0$. For simplicity set $C S_{M}=0$ so that the middle term in (8) can be ignored. Since the expected industry value term reaches an interior maximum at $\widehat{K}$, its derivative at this point vanishes. The left derivative of social welfare at $\widehat{K}$ is then equal to the left derivative of the consumer surplus from imitation term. In an attrition regime and near $\widehat{K}$ (for $K \in(\widetilde{K}, \widehat{K})$ ), this term has two parts that correspond to innovator investment threshold realizations below $Y_{S}^{\prime}$ (in which case imitator investment occurs at $Y_{F}$, see Figure 2) or above $Y_{S}$ (in which case imitation immediately follows innovation). Accounting for the equilibrium distribution of the imitation threshold $\max \left\{\widetilde{Y}_{I}, Y_{F}\right\}$, the consumer surplus from imitation is

$$
\frac{\left(\mathrm{CS}_{D}-\mathrm{CS}_{M}\right)}{r-\alpha}\left[Y_{F}\right]^{-(\beta-1)} y^{\beta}\left(G_{\wedge}\left(Y_{S}\right)+\int_{Y_{S}}^{\infty}\left(Y_{F} / s\right)^{\beta-1} d G_{\wedge}(s)\right) .
$$

At $\widehat{K}$ the equilibrium distribution $G_{\wedge}$ converges to a degenerate distribution at $Y_{L}$ and the integral term vanishes. Just to the left of $\widehat{K}$, changes in $K$ therefore have a negligible effect on the distribution of entry thresholds and only the direct effect on $Y_{F}$ matters, which is sufficient to establish the desired result.

Either type of local maximum (under attrition or preemption) can be a global maximum depending on the relative magnitude of the consumer surplus resulting from innovation and from imitation.

Proposition 3 If the timing of entry is regulated indirectly through an imitation cost instrument,

(i) either attrition or preemption may constitute a social optimum;

(ii) if the social optimum involves attrition, the cost of imitation is positive with $K^{*}>\widetilde{K}$ ("winnerpays-some");

(iii) if the social optimum involves preemption, innovation occurs at the threshold $Y_{P}^{*}=\psi Y_{L}$, $\psi \in[(\beta-1) / \beta, 1]$; for $\beta$ large enough a perpetual monopoly is optimal, that is $K^{*}=\infty$ (winnertake-all). ${ }^{14}$

The upshot of Proposition 3 is that there is no "one size fits all" prescription with respect to balancing the incentives of innovating and imitating firms, suggesting that policy is best determined on a case by case basis according to a number of industry conditions as is generally

\footnotetext{
${ }^{14}$ See Section A.6 for a characterization of $\psi:=\max \left\{\frac{\beta-1}{\beta},\left(\frac{\mathrm{CS}_{D}-\mathrm{CS}_{M}}{\pi_{D}}+\frac{2}{\beta}\right) /\left(\frac{\mathrm{CS}_{D}}{\pi_{D}}-\frac{\beta-1}{\beta} \frac{\mathrm{CS}_{M}}{\pi_{M}}+\frac{2}{\beta}\right)\right\}$. Note that in contrast with the optimal preemption thresholds that we derive explicitly, in preemption games these thresholds generally do not have analytic expressions.
} 
understood in the literature on IP protection levels. The proposition is nevertheless informative in a number of ways.

Part (ii) offers some practical guidance and is especially relevant insofar as some researchers have argued for the abolition of patents altogether (e.g. Boldrin and Levine [3]). Our model points to the fact that such an abolition is desirable only to the extent that the natural cost of imitation is sufficiently high, i.e. that $K \geq \widetilde{K}$ without IP protection. This lower bound on imitation cost has a straightforward characterization, in that industry conditions must be such that an innovator has a positive ex-ante probability of earning a monopoly profit rather than face immediate imitation with certainty, that is to say it must have some chance of avoiding the situation described by Scherer (see footnote 4 above). This lower bound can be used, for example, to assess the initiatives that have been taken in the pharmaceutical industry in order to reduce the relative cost of imitation and encourage generic competition. In low- and middle-income countries, often characterized by a rapidly expanding and highly uncertain demand which makes attrition more likely to prevail, optimal social welfare in the local market may involve attrition but in all cases requires that a minimal level of IP protection be maintained so that there remains a window of market sizes in which an innovator entering sufficiently early is incentivized by a period of monopoly profits.

Part (iii) establishes that in other circumstances where firms sufficiently discount the future, that is when demand growth and volatility are sufficiently low (recall that $\partial \beta / \partial \alpha<0$ and $\partial \beta / \partial \sigma<$ $0)$, the optimal form of preemption is a winner-take-all contest. In that case, the greatest amount of preemption that the social planner can induce $\left(K^{*}=\infty\right.$, a corner solution derived in Appendix A.6) implies that a single firm is active ex-post. Its investment threshold, $Y_{P}\left(K^{*}\right)=(r-\alpha) I / \pi_{M}$ corresponds to the Marshallian investment threshold and is determined by the threat of potential entry by the other firm, which in equilibrium never enters (as $\lim _{K \rightarrow \infty} Y_{F}=\infty$ ).

Even in the absence of a universal policy prescription certain industry characteristics can play a role in determining optimal protection levels. The following corollary provides two intuitive applications of part $(i)$ of the previous proposition.

\section{Corollary 3 In a constrained social optimum:}

(i) if there is perfect price discrimination under monopoly $\left(C S_{M}=0\right)$, attrition is socially optimal; (ii) if there is collusion in the product market $\left(C S_{D}+2 \pi_{D}=C S_{M}+\pi_{M}\right)$, preemption is socially optimal.

In part $(i)$ of the corollary, the innovator can price discriminate perfectly, so it does not contribute to the consumer surplus. Therefore, imitation is needed for consumers to benefit from 
the sequence of investments and an optimal protection regime favors imitation incentives under the constraint that innovation, as a necessary condition for imitation, must be incentivized as well. At the opposite extreme is the situation of part $(i i)$, where imitator entry does not change the instantaneous welfare because of collusion in the product market. Then only innovation contributes to social welfare, and a high -though not necessarily infinite- imitation cost is needed to incite early enough investment in a preemption regime.

Aside from such specific cases as those above, the absence of a closed form in the preemption range renders the characterization of optimal welfare more complex. By making an additional restriction however, we are able to obtain a general result relating optimal welfare to demand characteristics. Suppose therefore that the static entry incentive is socially excessive $\left(\pi_{D} \geq\left(\mathrm{CS}_{D}+2 \pi_{D}\right)-\left(\mathrm{CS}_{M}+\pi_{M}\right)\right)$. To provide a rationale for this restriction, recall that in a static setting with symmetric firms and homogeneous goods, Mankiw and Whinston [25] show that there is excess entry in an industry if total output increases whereas individual outputs decrease in the number of firms (the business-stealing effect) and argue that these assumptions characterize a broad range of common models of oligopoly. In our dynamic setting, this assumption is useful because it allows us to bound the welfare associated with the imitator's entry by the expected value of a duopoly firm, and hence by $\left.E V\left(Y_{L}, Y_{L}\right)\right|_{K=\widehat{K}}$ (according to Proposition 2), so as to establish the following.

Proposition 4 Suppose that the static private entry incentive is socially excessive. Then, in a constrained social optimum preemption is optimal if

$$
\frac{\mathrm{CS}_{M}}{\pi_{M}} \geq \Omega(\beta) \cdot{ }^{15}
$$

As the right-hand term is decreasing in $\beta$ with $\lim _{\rightarrow \infty} \Omega(\beta)=0$, the condition (10) is satisfied for any given demand specification if there is sufficient discounting, as occurs if industry growth and volatility are sufficiently low. This condition provides a rigorous foundation for the idea that IP protection should be significant in such markets.

We conclude this section by applying Proposition 4 to two common homogeneous goods oligopoly specifications.

Example Suppose that the product market is characterized by a linear inverse demand $P=A-B Q$, $A, B>0$ and that firms have constant unit variable cost $c$. Then $\mathrm{CS}_{M} / \pi_{M}=0.5$ and evaluating $\Omega(\beta)$ establishes that $(10)$ is satisfied for $\beta \geq 3.14$. If the product market is characterized instead

\footnotetext{
${ }^{15}$ See Section A.7 for a derivation of $\Omega(\beta):=2 /\left(\left(\beta^{\beta} /(\beta-1)^{\beta-1}\right)-\beta\right)$.
} 
by a constant elasticity inverse demand $P=A Q^{-1 / \varepsilon}, A>0$ and $\varepsilon>1$, then straightforward calculations establish $\mathrm{CS}_{M} / \pi_{M}=1 /(\varepsilon-1)$ so (10) is satisfied for any $\beta$ if demand is sufficiently elastic (if $\varepsilon \geq 1+(1 / \Omega(\beta))$ ).

\section{Endogenous imitation cost, buyout and licensing}

In this section, we discuss further real-world aspects of innovation and imitation and show how they fit into the framework of the previous sections. One aspect is the ability of an innovating firm to raise the entry barrier of the imitator, either by developing a product that is more costly to reverse engineer or by strengthening the patentability of its product. Another aspect is contracting between the innovator and the imitator, which can take the form of buyout of the rival firm or of a technology transfer that reduces the follower's imitation cost. From a formal standpoint these two extensions both add an intermediate decision layer to the investment game in the monopoly phase, once the innovator's entry has occurred and before the imitator invests. By raising the standalone value of the innovating firm, both extensions tend to favor first-mover advantage and the emergence of preemption regimes although the implications for imitation timing and welfare largely differ.

\subsection{Endogenous imitation cost}

Suppose that the innovating firm may rely on a variable amount of either technical or legal protection in order to determine the imitation cost of a subsequent entrant. In case of technical protection, imitation cost is determined in part by the cost of reverse engineering, which can be raised by increasing product complexity. For example an innovating firm can expend effort to render its product more difficult to disassemble, or even add misleading complexity (Samuelson and Scotchmer [30]). In the case of legal protection, the imitation cost is related to patent breadth with wider patents implying higher costs for inventing around so as to develop a non-infringing imitation, and firms moreover may decide to pursue patent protection more or less aggressively, as is the case for pharmaceutical firms as discussed in Section 2.

Such behavior is incorporated into the model of investment by supposing that at the time of its investment at a threshold $Y_{i}$ an innovating firm chooses to expend an additional irrecuperable cost, which we denote by $\rho$, that raises the imitating firm's fixed cost by an amount $f(\rho)$. The increase in imitation cost is assumed to be instantaneous and the function $f$ is taken to twice differentiable with $f^{\prime}>0, f^{\prime \prime} \leq 0$ as well as $f(0)=0$ and $\lim _{\rho \rightarrow 0} f^{\prime}(\rho)=\infty$. The investment 
costs of the innovator and imitator are redefined as $I(\rho):=I_{0}+\rho$ and $K(\rho):=K_{0}+f(\rho)$ where $I_{0}$ and $K_{0}$ represent then baseline values where no cost-raising expenditure is undertaken.

Proceeding by backward induction, in the continuation stage the imitator payoff $F(Y)$ and standalone threshold $Y_{F}$ are accordingly functions of the innovator's effort through $K(\rho)$. At the moment of innovation therefore, an innovator entering at the threshold $Y_{i}$ faces the decision problem

$$
\max _{\rho \in \mathbb{R}_{+}} L_{\mathrm{E}}\left(Y_{i}, \rho\right):=\left(\frac{\pi_{M}}{r-\alpha} Y_{i}-I_{0}-\rho\right)\left(\frac{y}{Y_{i}}\right)^{\beta}-\frac{\pi_{M}-\pi_{D}}{r-\alpha} \frac{y^{\beta}}{\left[\max \left\{Y_{i}, Y_{F}(\rho)\right\}\right]^{\beta-1}} .
$$

Let $\rho^{*}\left(Y_{i}\right)$ denote the solution to this problem. At an interior solution, $Y_{F}\left(\rho^{*}\right)>Y_{i}$ and $\rho^{*}$ satisfies

$$
\beta\left(\frac{\pi_{M}}{\pi_{D}}-1\right) f^{\prime}\left(\rho^{*}\right)=\left(\frac{Y_{F}\left(\rho^{*}\right)}{Y_{i}}\right)^{\beta}
$$

A straightforward comparative static argument establishes that the optimal cost-raising effort is increasing in the investment threshold and decreasing in the baseline imitation cost. The latter property is in line with the biopharmaceutical industry case discussed in Section 2, where firms typically place greater reliance on patenting in the medications segment in which natural entry barriers are low than in the vaccines segment.

To proceed further we focus on the situation where $K_{0} \geq \widehat{K}$ so the investment game is naturally preemptive. ${ }^{16}$ Allowing the cost of imitation to be endogenous results in a higher leader payoff $L_{\mathrm{E}}\left(Y, \rho^{*}(Y)\right)$ and a lower follower payoff $F_{\mathrm{E}}\left(Y, \rho^{*}(Y)\right)=\left.F(Y)\right|_{K=K_{0}+\rho^{*}(Y)}$ that when this cost is exogenous. This makes the investment game more preemptive. Since equilibrium payoffs are pegged to the follower value under preemption, firms have a lower expected value in equilibrium. To avoid this penalizing outcome firms would prefer to both commit ex-ante not to exert any cost-raising effort in case they happen to lead the investment process. One way to achieve such a commitment is by agreeing to an open or common technological standard.

Proposition 5 If the cost of imitation is endogenous and the investment game is naturally preemptive firms benefit from agreeing ex-ante to a common standard.

\footnotetext{
${ }^{16}$ This restriction is not necessarily strictly speaking necessary for the analysis but relates specifically to optimal stopping. If the innovation threshold is high enough, corner solutions $\rho^{*}=0$ arise that result in a kink of the function $L_{\mathrm{E}}$. In such cases the threshold strategies we assume firms to follow needn't correspond to optimal investment behavior. Under preemption however such high innovation thresholds occur only off the equilibrium path.
} 


\subsection{Buyout and licensing}

Contracting in a variety of forms, ranging from joint ventures and different types of licensing contracts to acquisitions and pay-for-delay agreements, generally plays an important role in innovation decisions. These different measures have contrasting effects on investment incentives that can be readily sketched out within our framework. Suppose that firms have the opportunity to contract only once to transfer either asset or technology ownership in exchange for a lump sum transfer $(\varphi)$ from the innovating firm to the imitating firm, and assume for simplicity that the contract is written by the innovator who detains all bargaining power.

Because of the efficiency effect $\left(\pi_{M}>2 \pi_{D}\right)$, it is profitable in principle for an innovator to pay for its rival not to subsequently enter the market, by buying it out if it can or engaging in a limiting case of a pay-for-delay agreement. Proceeding by backward induction, in the continuation phase that begins when innovation occurs at a threshold $Y_{i}$, the remaining firm has an expected payoff $F\left(Y_{i}\right)$. At the moment the first firm innovates, the rival's continuation payoff constitutes a participation constraint for any contract that the innovator offers. The innovating firm therefore offers a transfer $\varphi^{*}\left(Y_{i}\right)=C\left(Y_{i}\right)$ when it invests whose value at $t=0$ is $F(Y)$ and the leader payoff becomes

$$
L_{\mathrm{B}}(Y):=\left(\frac{\pi_{M}}{r-\alpha} Y-I\right)\left(\frac{y}{Y}\right)^{\beta}-F(Y)
$$

The effect of allowing buyouts on the investment game is intuitive. In comparison with the case where they are ruled out, the follower payoff is unchanged whereas the leader payoff is at least as large rendering preemption more likely. It can be shown that all else equal the magnitude of the impact of the buyout option on the leader payoff depends on the strength of the efficiency effect, and if the latter is sufficiently strong or in industries with sufficiently high demand growth or volatility (specifically if $\pi_{M} / \pi_{D} \geq \beta+1$ ) attrition does not occur for any level of $K$.

Whether the possibility of buyout runs in the interest of firms or not depends on the cost of imitation. Under preemption expected profits are pegged to the follower value and therefore unaffected by the possibility of buyouts, whereas if the industry functions naturally under attrition $(K<\widehat{K})$ they are pegged to the leader value and increase if the possibility of buyouts is introduced, so that one would expect a more active market for acquisitions to develop in such industries, and all the more so if demand growth and volatility are high.

If a buyout is not possible an innovator must contend with follower entry but can recoup revenue from the imitator's investment by setting an appropriate license fee. Suppose that $K=$ $K_{0}+K_{I}$ where $K_{0}$ is an incompressible level of imitation cost reflecting such items as distribution 
and marketing expenses and $K_{I}$ denotes the part of the imitator's product development cost that can be eliminated by a technology transfer from the innovator. Because licensing does not allow the innovator to push back the moment of imitation, the optimal policy is to set the maximum license fee at the moment of imitation consistent with the participation constraint, $\varphi^{*}=-K_{I}$.

Proceeding by backward induction, the expected revenue from licensing constitutes an additional term and raises the leader payoff which becomes

$$
L_{\mathrm{L}}(Y):=\left(\frac{\pi_{M}}{r-\alpha} Y-I\right)\left(\frac{y}{Y}\right)^{\beta}-\left(\frac{\pi_{M}-\pi_{D}}{r-\alpha} \max \left\{Y, Y_{F}\right\}-K_{I}\right)\left(\frac{y}{\max \left\{Y, Y_{F}\right\}}\right)^{\beta} .
$$

As the leader payoff shifts up to the left of $Y_{F}$ while leaving the follower payoff function unchanged, the investment game is more preemptive with licensing as in the case of buyouts. However whereas the welfare consequences of introducing buyouts are ambiguous (firms weakly benefit and the consumer surplus from innovation increases because product innovation occurs earlier, but the consumer surplus from imitation is eliminated), the welfare consequences of licensing are unambiguously positive. The visible effect of licensing is of course the corresponding reduction in the duplication of $R \& D$ efforts, but the indirect benefit (beyond any increase in firm value that may occur if the industry functions initially under attrition) stems from the resulting acceleration of innovation which raises consumer surplus.

Thus,

Proposition 6 With contracting between the innovator and the imitator $(i)$ buyouts are the preferred instrument of an innovator and raise industry profit if $K<\widehat{K}$ whereas (ii) licensing is Pareto improving.

\section{Conclusion}

We have sought to develop a framework to study the allocation of resources to innovation and to imitation that explicitly incorporates interrelated investment decisions under uncertainty by imperfectly competitive firms. As compared with the classic literature on patent breadth, by endogenizing the time at which innovation and imitation occur we are able to highlight a novel channel through which IP protection levels act upon welfare through their effect on the dynamic competition between firms.

The main message that emerges from our analysis is a broadly familiar one insofar as we find that IP protection levels must be sufficiently high to provide appropriate incentives for innovation. 
By integrating the theory of investment under uncertainty into the analysis of innovation incentives we are able to sharpen this general perspective by pinpointing specific market characteristics that correspond to key determinants of investment, and which provide a grounding for strong IP protection in circumstances that seem most likely to be present in mature industries. In such industries then we find that the barriers to imitation should be sufficiently high so as to render dynamic competition between firms preemptive, and if discounting is important enough it should take the form of a winner-take-all contest.

In those industries in which growth and volatility are sufficiently high, and which are those most typically associated with vibrant innovation, attrition may also be optimal so that the additional benefits of imitation resulting from greater product market competition do not arrive excessively late. Even then IP protection may be necessary if the cost of imitation is extremely low, in order to ensure that a firm that develops an imitative product as the winner of the attrition game nevertheless "pays some" and that an industry does not become mired in inefficient dynamics described by Scherer (1980).

Throughout our analysis we have sought to illustrate the theory with stylized facts pertaining to a specific industry, the biopharmaceutical industry, in which both innovation and the regulation of innovation play a central role. In the practice of antitrust and industrial policy decisions it is common to focus upon static product market characteristics. Those demand characteristic that we have sought to highlight though, demand growth and volatility, play at least as significant a role in determining the investment incentives and product development decisions, and as such should naturally underlie any determination of optimal IP protection levels.

\section{References}

[1] Arora A, Branstetter L,Chatterjee C (2008) Strong medicine: patent reform and the emergence of a research-driven pharmaceutical industry in India, Paper presented at the NBER conference, Location of Biopharmaceutical Activity, Savannah, GA, March 72008.

[2] Billette de Villemeur E, Ruble R, Versaevel B (2014) Investment timing and vertical relationships, International Journal of Industrial Organization 33:110-123.

[3] Boldrin M, Levine D (2013) The case against patents, Journal of Economic Perspectives $27(1): 3-22$. 
[4] Chevalier-Roignant B, Trigeorgis L (2011) Competitive Strategy: Options and Games, (Cambridge: MIT Press).

[5] Cohen W M, Nelson RR, Walsh JP (2000) Protecting their intellectual assets: appropriability conditions and why U.S. manufacturing firms patent (or not), NBER Working Paper No. 7552.

[6] Denicolò V (1996) Patent races and optimal patent breadth and length, Journal of Industrial Economics 44(3):249-265.

[7] Denicolò V, Franzoni L (2010) On the winner-take-all principle in innovation races, Journal of the European Economic Association 8(5):1133-1158.

[8] Dixit A, Pindyck R (1994) Investment under Uncertainty, (Princeton: Princeton University Press).

[9] Encaoua D, Guellec D, Martinez C (2006) Patent systems for encouraging innovation: Lessons from economic analysis, Research Policy 35(9):1423-1440.

[10] Femminis G, Martini G (2011) Irreversible investment and R\&D spillovers in a dynamic duopoly, Journal of Economic Dynamics and Control 35(7):1061-1090.

[11] Fudenberg D, Tirole J (1985) Preemption and rent equalization in the adoption of new technology, Review of Economic Studies 52(3):383-401.

[12] Gallini N (1992) Patent policy and costly imitation, RAND Journal of Economics 23(1):52-63.

[13] Gilbert R, Shapiro C (1990) Optimal patent length and breadth, RAND Journal of Economics 21(1):106-112.

[14] Grabowski HG, DiMasi JA, Long G, (2015) The roles of patents and research and development incentives in biopharmaceutical innovation, Health Affairs 34(2):302-310.

[15] Green J, Scotchmer S (1995) On the division of profit in sequential innovation, RAND Journal of Economics 26(1):20-33.

[16] Guey Chuen P, Huan-Yao L, Yee-Shin L, Kulkanya C (2011) Dengue vaccines: challenge and confrontation,World Journal of Vaccines 1:109-130.

[17] Hendricks K, Weiss A, Wilson C (1988) The war of attrition in continuous time with complete information, International Economic Review 29(4):663-680. 
[18] Henry E (2010) Runner-up patents: is monopoly inevitable?, Scandinavian Journal of Economics 112(2):417-440.

[19] Henry E, Ruiz-Aliseda F (2015) Keeping secrets: the economics of access deterrence, mimeo.

[20] Hoen E, Berger J, Calmy A, Moon S (2011) Driving a decade of change: HIV/AIDS, patents and access to medicines for all, Journal of the International AIDS Society 14:15-26.

[21] Hoppe H (2000) Second-mover advantages in the strategic adoption of new technology under uncertainty, International Journal of Industrial Organization 18:315-338.

[22] Katz M, Shapiro C (1987) R and D rivalry with licensing or imitation, American Economic Review 77(3):402-420.

[23] Klemperer, P (1990) How bread should the scope of patent protection be?, Rand Journal of Economics 21(1):113-130.

[24] La Manna M, MacLeod R, de Meza D (1989) The case for permissive patents, European Economic Review 33(7):1427-43.

[25] Mankiw G, Whinston M (1986) Free entry and social inefficiency, RAND Journal of Economics $17(1): 48-58$.

[26] Maurer SM, Scotchmer S (2002) The independent invention defence in intellectual property, Economica 69(276):535-547.

[27] Mukherjee A, Pennings E (2004) Imitation, patent protection, and welfare, Oxford Economic Papers 56(4):715-733.

[28] Reinganum J (1981) On the diffusion of a new technology: a game theoretic approach, Review of Economic Studies 48(3):395-405.

[29] Riedel F, Steg J-H (2017) Subgame-perfect equilibria in stochastic timing games, forthcoming Journal of Mathematical Economics

[30] Samuelson P, Scotchmer S (2002) The law and economics of reverse engineering, Yale Law Journal 111:1575-1663.

[31] Scherer F, Watal J (2002) Post-TRIPS options for access to patented medicines in developing countries, Journal of International Economic Law 5(4):913-939. 
[32] Thijssen J (2013) Game theoretic real options and competition risk, in: Bensoussan A, Peng S, Sung J (eds.), Real Options, Ambiguity, Risk and Insurance (Amsterdam: IOS Press).

[33] Thijssen J, Huisman K, Kort P (2006) The effects of information on strategic investment and welfare, Economic Theory 28(2):399-424.

[34] Thijssen J, Huisman K, Kort P (2012) Symmetric equilibrium strategies in game theoretic real option models, Journal of Mathematical Economics 48(4):219-225.

[35] Wilson P (2010) Giving developing countries the best shot: An overview of vaccine access and R\&D, Oxfam International, 28 pages.

[36] Witty A (2010) New strategies for innovation in global health: A pharmaceutical industry perspective, Health Affairs 30(1):118-126.

\section{A Proofs and derivations}

\section{A.1 Continuation payoff $C$}

Once the rival firm has invested at $Y_{t}=y$ any remaining firm holds a standard growth option (Dixit and Pindyck [8]) whose value is obtained by solving an optimal stopping problem

$$
C(y)=\sup _{\tau \geq t} \mathbb{E}_{y}\left(\int_{\tau}^{\infty} \pi_{D} Y_{s} e^{-r s} d s-K e^{-r \tau}\right) .
$$

From the Hamilton-Jacobi-Bellman equation it follows that the value function $C(y)$ satisfies

$$
r C(y) d t=\mathbb{E}_{y} d C(y)
$$

and expanding the right-hand side using Itô's lemma yields the ordinary differential equation that $C(y)$ solves in the continuation region $\left(0, Y_{F}\right)$,

$$
r C=\alpha y C^{\prime}+\frac{1}{2} \sigma^{2} y^{2} C^{\prime \prime},
$$

along with boundary and smooth pasting conditions

$$
\begin{gathered}
C(0)=0 \\
C\left(Y_{F}\right)=\frac{\pi_{D}}{r-\alpha} Y_{F}-K \\
C^{\prime}\left(Y_{F}\right)=1 .
\end{gathered}
$$


The function $C(y)=A_{1} y^{\beta_{1}}+A_{2} y^{\beta_{2}}$ is a candidate solution. The associated fundamental quadratic is $0.5 \sigma^{2} \beta(\beta-1)+\beta \alpha-r=0$. It has two roots of which only

$$
\beta=\frac{1}{2}-\frac{\alpha}{\sigma^{2}}+\sqrt{\left(\frac{\alpha}{\sigma^{2}}-\frac{1}{2}\right)^{2}+\frac{2 r}{\sigma^{2}}}
$$

is positive. Setting $\beta_{1}=\beta$ and $A_{2}=0$ to satisfy the first boundary condition, it follows from the remaining conditions that

$$
Y_{F}=\frac{\beta}{\beta-1} \frac{r-\alpha}{\pi_{D}} K
$$

and that $A_{1}=(1 / \beta)\left[Y_{F}\right]^{-(\beta-1)}$ which yields the expression for $A_{C}$ in the text.

\section{A.2 Leader, follower and simultaneous investment payoff functions $L, F$ and $M$}

\section{A.2.1 Derivation of $L, F$ and $M$}

The expression (3) is obtained from the definition of $L(Y)$ by the following steps. Since the rival's entry can equivalently be viewed as a negative shock to the firm's perpetual stream of monopoly profits starting at time $\tau\left(\max \left\{Y, Y_{F}\right\}\right)$,

$$
\begin{aligned}
\mathbb{E}_{y}\left(\int_{\tau(Y)}^{\tau\left(\max \left\{Y, Y_{F}\right\}\right)}\right. & \left.\pi_{M} Y_{s} e^{-r s} d s-e^{-r \tau(Y)} I+\int_{\tau\left(\max \left\{Y, Y_{F}\right\}\right)}^{\infty} \pi_{D} Y_{s} e^{-r s} d s\right) \\
= & \mathbb{E}_{y}\left(\int_{\tau(Y)}^{\infty} \pi_{M} Y_{s} e^{-r s} d s-e^{-r \tau(Y)} I-\int_{\tau\left(\max \left\{Y, Y_{F}\right\}\right)}^{\infty}\left(\pi_{M}-\pi_{D}\right) Y_{s} e^{-r s} d s\right) .
\end{aligned}
$$

Moving the constant terms outside the integrals and using $\mathbb{E}_{y} e^{-r \tau(Y)} d s=(y / Y)^{\beta}$, the martingale property of Brownian motion and $\mathbb{E}_{y} \int_{0}^{\infty} Y_{s} e^{-r s} d s=(y /(r-\alpha))$,

$$
\begin{aligned}
\mathbb{E}_{y}\left(\int_{\tau(Y)}^{\infty} \pi_{M} Y_{s} e^{-r s} d s-e^{-r \tau(Y)} I-\right. & \left.\int_{\tau\left(\max \left\{Y, Y_{F}\right\}\right)}^{\infty}\left(\pi_{M}-\pi_{D}\right) Y_{s} e^{-r s} d s\right) \\
& =\left(\frac{\pi_{M}}{r-\alpha} Y-I\right)\left(\frac{y}{Y}\right)^{\beta}-\frac{\pi_{M}-\pi_{D}}{r-\alpha} \frac{y^{\beta}}{\left[\max \left\{Y, Y_{F}\right\}\right]^{\beta-1}} .
\end{aligned}
$$

The expression for the follower payoff (4) results from discounting the continuation payoff $C$, which is realized at the threshold $\max \left\{Y, Y_{F}\right\}$, to the current time and level of the stochastic shock. Finally the simultaneous payoff (5) is derived similarly to (3), noting that the flow profit upon investment is $\pi_{D}$ instead of $\pi_{M}$ and that there is no follow-on entry so the bound $\infty$ can be substituted for $\tau\left(\max \left\{Y, Y_{F}\right\}\right)$ in the first integral and the second integral dropped altogether. 


\section{A.2.2 Behavior of $L, F$ and $M$}

The most straightforward continuation payoffs to study are $M$ and $F$, and these are discussed first before studying $L$.

First, $M(Y)=\left(\left(\pi_{D} /(r-\alpha)\right) Y-I\right)(y / Y)^{\beta}$ is quasiconcave in $Y$ over $(y, \infty)$ with a maximum at $Y_{S}:=(\beta(r-\alpha) I) /\left((\beta-1) \pi_{D}\right)$ such that $Y_{S}>y$ by the assumption on $Y_{0}$, and $\lim _{Y \rightarrow \infty} M(Y)=0$. Observe that $M(Y)$ is independent of $K$.

Next, it is convenient to express $F(Y)$, which is the value at $t=0$ of perceiving the continuation payoff $C(Y ; K)$, as

$$
F(Y)= \begin{cases}A_{C} y^{\beta} & Y<Y_{F} \\ \left(\frac{\pi_{D}}{r-\alpha} Y-K\right)\left(\frac{y}{Y}\right)^{\beta} & , \quad Y \geq Y_{F}\end{cases}
$$

Note that $F$ is differentiable at $Y_{F}$. If $K<\left((\beta-1) \pi_{D} y\right) /(\beta(r-\alpha)), Y_{F}<y$ and $F$ is strictly decreasing in $Y$ over $(y, \infty)$. Otherwise $F$ is constant over $\left[y, Y_{F}\right]$ and decreasing over $\left(Y_{F}, \infty\right)$ with $\lim _{Y \rightarrow \infty} F(Y)=0$.

An increase in $K$ has two principal effects on $F(Y): Y_{F}$ increases so the graph of $F$ is flat over a longer interval and $F(Y)$ increases for all $K$ so the graph of $F$ shifts upward. The comparison of $M(Y)$ and $F(Y)$ is straightforward: $Y_{S}<(>) Y_{F}$ if and only if $K>(<) I$ and if $K<I$ then $M(Y)<F(Y)$ for all $Y$ whereas if $K>I$ then $M(Y)>F(Y)$ over a half-line $(a, \infty)$ where $a \in\left(y, Y_{S}\right)$.

Finally, the leader payoff $L(Y)$ is typically defined in parts according to whether $Y$ is greater or smaller than $Y_{F}$. If $K<\left((\beta-1) \pi_{D} y\right) /(\beta(r-\alpha))$, necessarily $Y_{F}<Y$ and follower investment is immediate at any threshold, so that $L(Y)=M(Y)$ since the leader firm does not enjoy a monopoly phase. Thus

$$
L(Y)=\left\{\begin{array}{ll}
\left(\frac{\pi_{M}}{r-\alpha} Y-I\right)\left(\frac{y}{Y}\right)^{\beta}-\frac{\pi_{M}-\pi_{D}}{r-\alpha} Y_{F}\left(\frac{y}{Y_{F}}\right)^{\beta} & , \quad Y<Y_{F} \\
M(Y) & , \quad Y \geq Y_{F}
\end{array} .\right.
$$

Note that $L$ is continuous with a kink at $Y_{F}$. The first part of $L$ is quasiconcave in $Y$ with a maximum at $Y_{L}:=(\beta(r-\alpha) I) /\left((\beta-1) \pi_{M}\right)$ such that $Y_{L}>y$ by assumption.

An increase in $K$ has two principal effects on $L(Y): Y_{F}$ increases reducing the range over which the graphs of $L$ and $M$ overlap, and the first part of $L(Y)$ increases shifting the graph of $L$ upward (a level effect). Because $\pi_{M}>\pi_{D}, Y_{L}<Y_{S}$ for all $K$ whereas $Y_{L}<(>) Y_{F}$ if and only if $I<(>)\left(\pi_{M} / \pi_{D}\right) K$. Given the ranking of $Y_{S}$ and $Y_{F}$ discussed above it follows that whenever 
$K \leq I, L$ has two local maxima $\left(Y_{L}\right.$ and $\left.Y_{S}\right)$. It is shown in the next subsection that there exists a critical threshold $\widetilde{K}<I$ such that $Y_{L}\left(Y_{S}\right)$ is a global maximum of $L$ if and only if $K>(<) \widetilde{K}$.

The relationship between $L$ and $M$ is straightforward as $L$ is greater than $M$ over $\left(y, Y_{F}\right)$ and the two functions coincide over $\left[Y_{F}, \infty\right)$. To compare $L$ and $F$ first note that for sufficiently low values of $K, L<F$ for all $Y$, but otherwise the two functions may intersect. It is also shown in the next subsection that there exists a critical threshold $\widehat{K}<I$ such that $i) Y_{L}$ is a global maximum of $L$ and $i$ ) both $L$ and $F$ attain the same maximum value at $Y_{L}$ and $Y_{F}$ respectively. Above this threshold, direct calculations establish that either $K<I$ in which case $F>L$ over an interval $\left(a^{\prime}, b^{\prime}\right) \subseteq\left(y, Y_{F}\right)$ or $K \geq I$ in which case $L \geq F$ for all $Y$.

\section{A.2.3 Critical thresholds $\widetilde{K}$ and $\widehat{K}$}

The threshold $\widetilde{K}$ solves $L\left(Y_{L}\right)=M\left(Y_{S}\right)$. If $\widetilde{K}<\left(\pi_{D} / \pi_{M}\right) I$ then $\max \left\{Y_{L}, Y_{F}\right\}=Y_{L}$ so after substituting, $L\left(Y_{L}\right)=\left(\left(\pi_{D} /(r-\alpha)\right) Y_{L}-I\right)\left(y / Y_{L}\right)^{\beta}=M\left(Y_{L}\right)<M\left(Y_{S}\right)$ since $Y_{S}$ is the unique global maximizer of $M$. Hence it must be that $\widetilde{K} \geq\left(\pi_{D} / \pi_{M}\right) I$, and therefore satisfies

$$
\left(\frac{\pi_{M}}{r-\alpha} Y_{L}-I\right)\left(\frac{y}{Y_{L}}\right)^{\beta}-\frac{\pi_{M}-\pi_{D}}{r-\alpha} Y_{F}\left(\frac{y}{Y_{F}}\right)^{\beta}=\left(\frac{\pi_{D}}{r-\alpha} Y_{S}-I\right)\left(\frac{y}{Y_{S}}\right)^{\beta} .
$$

Multiplying both sides by $\left(Y_{F} / y\right)^{\beta}$ (note that $Y_{F} / Y_{L}=\left(\pi_{M} / \pi_{D}\right)(\widetilde{K} / I)$ and $Y_{F} / Y_{S}=\widetilde{K} / I$ here) and substituting for remaining $Y_{L}, Y_{F}$ and $Y_{S}$ terms yields

$$
\frac{1}{\beta-1}\left(\frac{\pi_{M}}{\pi_{D}}\right)^{\beta}[\widetilde{K}]^{\beta} I^{-(\beta-1)}+\frac{\beta}{\beta-1}\left(1-\frac{\pi_{M}}{\pi_{D}}\right) \widetilde{K}=\frac{1}{\beta-1}[\widetilde{K}]^{\beta} I^{-(\beta-1)}
$$

which has a unique positive solution,

$$
\widetilde{K}=\left(\frac{\beta\left(\frac{\pi_{M}}{\pi_{D}}-1\right)}{\left(\frac{\pi_{M}}{\pi_{D}}\right)^{\beta}-1}\right)^{\frac{1}{\beta-1}} I .
$$

The threshold $\widehat{K}$ solves $L\left(Y_{L}\right)=L\left(Y_{F}\right)$. If $K \leq \widetilde{K}$ then as shown above $L\left(Y_{L}\right) \leq M\left(Y_{S}\right)$ whereas $M\left(Y_{S}\right)<L\left(Y_{F}\right)$. Hence it must be that $\widehat{K}>\widetilde{K}$ (and $\max \left\{Y_{L}, Y_{F}\right\}=Y_{F}$ ). This threshold therefore solves

$$
\left(\frac{\pi_{M}}{r-\alpha} Y_{L}-I\right)\left(\frac{y}{Y_{L}}\right)^{\beta}-\frac{\pi_{M}-\pi_{D}}{r-\alpha} Y_{F}\left(\frac{y}{Y_{F}}\right)^{\beta}=\frac{y^{\beta}}{\beta\left[Y_{F}\right]^{(\beta-1)}}
$$


Multiplying both sides by $\left(Y_{F} / y\right)^{\beta}$ (note that here $Y_{F} / Y_{L}=\left(\pi_{M} / \pi_{D}\right)(\widehat{K} / I)$ ) and substituting for remaining $Y_{L}$ and $Y_{F}$ terms yields

$$
\frac{I}{\beta-1}\left(\frac{\pi_{M}}{\pi_{D}}\right)^{\beta}[\widehat{K}]^{\beta} I^{-(\beta-1)}+\frac{\beta}{\beta-1}\left(1-\frac{\pi_{M}}{\pi_{D}}\right) \widehat{K}=\frac{\widehat{K}}{\beta-1} .
$$

which has a unique positive solution,

$$
\widehat{K}=\left(\frac{1+\beta\left(\frac{\pi_{M}}{\pi_{D}}-1\right)}{\left(\frac{\pi_{M}}{\pi_{D}}\right)^{\beta}}\right)^{\frac{1}{\beta-1}} I .
$$

\section{A.3 Proof of Proposition 1}

\section{A.3.1 Part $(i)$}

As discussed in the text and illustrated in Figures 1 and 2 there are two subcases to consider in order to establish this part of the proposition, $K \leq \widetilde{K}$ and $\widetilde{K}<K<\widehat{K}$.

$K \leq \widetilde{K}$ case This case corresponds to a standard attrition game over $\left(Y_{S}, \infty\right)$. It is therefore only necessary to verify that firms do not move in $\left[y, Y_{S}\right]$ with positive probability, in which case a specific form for the equilibrium distribution can be derived following standard arguments.

First, if $Y_{i} \in\left[y, Y_{S}\right), V\left(Y_{i}, Y_{j}\right)=L\left(Y_{i}\right)<M\left(Y_{S}\right)$ if $Y_{i}<Y_{j},=M\left(Y_{i}\right)<M\left(Y_{S}\right)$ if $Y_{i}=Y_{j}$, $=F\left(Y_{j}\right)$ if $Y_{i}>Y_{j}$, so $V\left(Y_{i}, Y_{j}\right) \leq V\left(Y_{S}, Y_{j}\right)$ for any $Y_{j}$ so that any $Y_{i}$ in this interval is a dominated strategy. If $Y_{i}=Y_{S}$ then it must be verified that players do not play an atom at this point in a symmetric equilibrium. But if player $j$ invests at $Y_{S}$ with probability $q>0$, by continuity of $L$ there exists $\varepsilon>0$ such that $\mathbb{E} V\left(Y_{S}, \widetilde{Y}_{j}\right)=q M\left(Y_{S}\right)+(1-q) L\left(Y_{S}\right)=M\left(Y_{S}\right)<$ $q F\left(Y_{S}+\varepsilon\right)+(1-q) L\left(Y_{S}+\varepsilon\right) \approx \mathbb{E} V\left(Y_{S}+\varepsilon, \widetilde{Y}_{j}\right)$.

Following Hendricks et al. [17] firms randomize investment triggers continuously over $\left[Y_{S}, \infty\right)$. To derive the unconditional equilibrium distribution assume that firm $j \neq i$ randomizes her investment trigger according to a distribution $G$ with density $g$. Firm $i$ 's expected payoff from investing at a threshold $Y_{i} \geq Y_{S}$ is

$$
\begin{aligned}
\int_{Y_{S}}^{\infty} V\left(Y_{i}, s\right) g(s) d s & =\int_{Y_{S}}^{Y_{i}} F(s) g(s) d s+\int_{Y_{i}}^{\infty} M\left(Y_{i}\right) g(s) d s \\
& =\int_{Y_{S}}^{Y_{i}} F(s) g(s) d s+(1-G(s)) M\left(Y_{i}\right) .
\end{aligned}
$$


Firm $i$ is willing to mix over investment thresholds if $\partial\left(\int_{Y_{S}}^{\infty} V\left(Y_{i}, s\right) g(s) d s\right) / \partial Y_{i}=0$ over $\left(Y_{S}, \infty\right)$, that is if $G$ is such that $[F(Y)-M(Y)] g(Y)=-M^{\prime}(Y)[1-G(Y)]$ for all $Y \in\left(Y_{S}, \infty\right)$. As the same condition holds for the other by symmetry the equilibrium distribution satisfies

$$
G_{a}(Y)=1-\exp \int_{Y_{S}}^{Y} \frac{M^{\prime}(s)}{F(s)-M(s)} d s
$$

so substituting for $F$ and $M$ the equilibrium distribution is

$$
G_{a}(Y)=\left\{\begin{array}{ll}
0 & Y<Y_{S} \\
1-\left(\frac{Y}{Y_{S}}\right)^{\beta \frac{I}{I-K}} \exp \left\{-\beta \frac{I}{I-K}\left(\frac{Y}{Y_{S}}-1\right)\right\}, & Y \geq Y_{S}
\end{array} .\right.
$$

$\widetilde{K}<K<\widehat{K}$ case As $L(Y)<F(Y)$ for all $Y$ firms engage in a war of attrition but since $L(Y)$ is decreasing over $\left(Y_{L}, Y_{S^{\prime}}\right)$, constant over $\left[Y_{S^{\prime}}, Y_{S}\right]$ and decreasing over $\left(Y_{S}, \infty\right)$ the war of attrition has a nonstandard form, where $Y_{S^{\prime}}$ denotes the unique solution in $\left[Y_{L}, Y_{F}\right]$ to the condition $L(Y)=M\left(Y_{S}\right)$.

The support of mixed strategies follows from an argument similar to above. Any threshold $Y_{i} \in\left(y, Y_{L}\right)$ is a strictly dominated strategy and no player puts positive probability on $Y_{L}$ in a symmetric equilibrium. Similarly investing at any threshold $Y_{i} \in\left(Y_{S^{\prime}}, Y_{S}\right)$ is strictly dominated by investing at $Y_{S}$ and no player invests with positive probability at $Y_{S}$ in a symmetric equilibrium. Investment thresholds are therefore continuously distributed over $\left[Y_{L} Y_{S^{\prime}}\right] \cup\left[Y_{S}, \infty\right)$.

Letting $G_{b}$ and $g_{b}$ denote the unconditional equilibrium distribution and density assume that firm $j \neq i$ randomizes her investment trigger. Firm $i$ is indifferent between investment thresholds if $\partial\left(\int_{Y_{S}}^{\infty} V\left(Y_{i}, s\right) g_{b}(s) d s\right) / \partial Y_{i}=0$ over $\left[Y_{L}, Y_{S^{\prime}}\right] \cup\left[Y_{S}, \infty\right)$, that is if $G_{b}$ is such that $[F(Y)-L(Y)] g_{b}\left(Y_{i}\right)=-L^{\prime}(Y)\left[1-G_{b}(Y)\right]$ for all $Y \in\left(Y_{L} Y_{S^{\prime}}\right)$ and $[F(Y)-M(Y)] g_{b}(Y)=$ $-M^{\prime}(Y)\left[1-G_{b}(Y)\right]$ for all $Y \in\left(Y_{S}, \infty\right)$. The former condition holds for

$$
\begin{aligned}
G_{a^{\prime}}(Y) & =1-\exp \int_{Y_{L}}^{Y} \frac{L^{\prime}(s)}{F\left(Y_{F}\right)-L(s)} d s \\
& =\frac{L\left(Y_{L}\right)-L(Y)}{F\left(Y_{F}\right)-L(Y)}
\end{aligned}
$$

while the latter condition is satisfied by $G_{a}$ so that the equilibrium distribution is

$$
G_{b}(Y)=\left\{\begin{array}{ll}
0 & \text { if } Y<Y_{L} \\
G_{a^{\prime}}(Y) & \text { if } Y_{L} \leq Y \leq Y_{S^{\prime}} \\
G_{a^{\prime}}\left(Y_{S^{\prime}}\right) & \text { if } Y_{S^{\prime}}<Y<Y_{S} \\
G_{a^{\prime}}\left(Y_{S^{\prime}}\right)+\left(1-G_{a^{\prime}}\left(Y_{S^{\prime}}\right)\right) G_{a}(Y) & \text { if } Y \geq Y_{S}
\end{array} .\right.
$$




\section{A.3.2 Part $(i i)$}

As discussed in the text and illustrated in Figures 3 and 4 there are two subcases to consider in order to establish this part of the proposition. It is simpler to begin with the more standard case $I \leq K$ before considering the case $\widehat{K}<K<I$.

$I \leq K$ case $\quad$ For $K \geq I, L\left(Y_{F}\right) \geq F\left(Y_{F}\right)$ so that there exists a unique $Y_{P}<Y_{F}$ such that $L(Y)=$ $F(Y)$. Over $\left(Y_{P}, Y_{F}\right)$, the preemption range, $L(Y)>F(Y)$. Following standard arguments over this range firms seek to enter before the rival and in equilibrium both firms invest at $Y_{P}$ which, by the tie-breaking rule, results in either firm investing at $Y_{P}$ with equal probability.

In preemption models, joint investment equilibria may also arise and it must be verified that this is not the case here. Investment at the optimal simultaneous investment threshold $Y_{S}$ by both firms results in a payoff $M\left(Y_{S}\right)$ and evaluating the ratio of payoffs,

$$
\frac{V\left(Y_{L}, Y_{S}\right)}{V\left(Y_{S}, Y_{S}\right)}=\frac{L\left(Y_{L}\right)}{M\left(Y_{S}\right)}=\left(\frac{\pi_{M}}{\pi_{D}}\right)^{\beta}-\beta\left(\frac{\pi_{M}}{\pi_{D}}-1\right)\left(\frac{I}{K}\right)^{\beta-1} .
$$

This ratio is increasing in $K$ and therefore bounded below by its value at $\widehat{K}$ (over the range of investment costs for which preemption occurs). Substituting $\widehat{K}$ for $K$ and simplifying gives $L\left(Y_{L}\right) / M\left(Y_{S}\right)=(I / \widehat{K})^{(\beta-1)}>1$. The best response to $Y_{j}=Y_{S}$ is thus $Y_{L}$ for all $K>\widehat{K}$ and simultaneous investment is not an equilibrium.

$\widehat{K}<K<I$ case For $\widehat{K}<K<I$, the condition $L(Y)=F(Y)$ has two roots $Y_{P}, Y_{P^{\prime}}$ with $Y_{P}<Y_{L}$ and $Y_{P^{\prime}} \in\left(Y_{L}, Y_{F}\right)$. For a given $Y>Y_{P^{\prime}}$ and any $Y_{j}, V\left(Y, Y_{j}\right) \leq F\left(Y_{F}\right)$ so playing beyond the preemption range $\left(Y_{P}, Y_{P^{\prime}}\right)$ is a dominated strategy. Over the preemption range $\left(Y_{P}, Y_{P^{\prime}}\right)$ firms preempt one another as in case 3 above and in equilibrium both firms invest at $Y_{P}$ which by the tie-breaking rule results in either firm investing at $Y_{P}$ with equal probability.

\section{A.3.3 Part $(i i i)$}

If $K=\widehat{K}$, then any $Y_{i}=Y_{L}$ and $Y_{j} \geq Y_{L}$ constitute an equilibrium as players are indifferent between the resulting leader and follower roles $\left(L\left(Y_{L}\right)=F\left(Y_{L}\right)\right)$, and of these equilibria only $\left(Y_{L}, Y_{L}\right)$ is symmetric. 


\section{A.4 Corollary 2}

To establish the comparative statics results the effects of $\pi_{M} / \pi_{D}$ and $\beta$ and on $\widehat{K}$ need to be determined. Evaluating the relevant partial derivatives and rearranging yields

$$
\frac{\partial \widehat{K}}{\partial\left(\pi_{M} / \pi_{D}\right)}=-\beta \frac{\frac{\pi_{M}}{\pi_{D}}-1}{\frac{\pi_{M}}{\pi_{D}}\left(1+\beta\left(\frac{\pi_{M}}{\pi_{D}}-1\right)\right)} \widehat{K}
$$

after rearrangement so $\partial \widehat{K} / \partial\left(\pi_{M} / \pi_{D}\right)<0$ directly, whereas

$$
\begin{aligned}
& \frac{\partial \widehat{K}}{\partial \beta}= \\
& \left(-\frac{\ln \frac{1+\beta\left(\left(\pi_{M} / \pi_{D}\right)-1\right)}{\left(\pi_{M} / \pi_{D}\right)^{\beta}}}{(\beta-1)^{2}}+\frac{1}{\beta-1} \frac{\left(\frac{\pi_{M}}{\pi_{D}}\right)^{\beta}}{\left(1+\beta\left(\frac{\pi_{M}}{\pi_{D}}-1\right)\right)} \frac{\left(\frac{\pi_{M}}{\pi_{D}}-1\right)\left(\frac{\pi_{M}}{\pi_{D}}\right)^{\beta}-\left(1+\beta\left(\frac{\pi_{M}}{\pi_{D}}-1\right)\right)\left(\frac{\pi_{M}}{\pi_{D}}\right)^{\beta} \ln \frac{\pi_{M}}{\pi_{D}}}{\left[\left(\frac{\pi_{M}}{\pi_{D}}\right)^{\beta}\right]^{2}}\right) \widehat{K} \\
& =\frac{-1}{(\beta-1)^{2}}\left(\ln \frac{1+\beta\left(\left(\pi_{M} / \pi_{D}\right)-1\right)}{\pi_{M} / \pi_{D}}-\frac{(\beta-1)\left(\left(\pi_{M} / \pi_{D}\right)-1\right)}{1+\beta\left(\left(\pi_{M} / \pi_{D}\right)-1\right)}\right) \widehat{K} .
\end{aligned}
$$

The sign of $\partial \widehat{K} / \partial \beta$ is the opposite of that of the (bracketed) middle term. Applying the logarithm inequality $\ln x>(x-1) / x$ for $x>0, x \neq 1$ with $x=\left(1+\beta\left(\left(\pi_{M} / \pi_{D}\right)-1\right)\right) /\left(\pi_{M} / \pi_{D}\right)$ yields

$$
\ln \frac{1+\beta\left(\left(\pi_{M} / \pi_{D}\right)-1\right)}{\pi_{M} / \pi_{D}}>\frac{(\beta-1)\left(\left(\pi_{M} / \pi_{D}\right)-1\right)}{1+\beta\left(\left(\pi_{M} / \pi_{D}\right)-1\right)}
$$

so $\partial \widehat{K} / \partial \beta<0$ and therefore $\partial \widehat{K} / \partial \alpha, \partial \widehat{K} / \partial \sigma>0$.

\section{A.5 Extended mixed strategies and dynamic representation of the timing game}

Suppose that the feasible firm investment strategies are taken to consist of investment times that are first-hitting times $\tau\left(Y_{i}\right):=\inf \left\{t \geq 0 \mid Y_{t} \geq Y_{i}\right\}$ consistently with the idea that managers determine optimal hurdle rates for investment at any point in time. Then the investment game is quasi-deterministic in nature in the sense that there is a one-to-one mapping between distributions of investment times and threshold strategies. We can then apply the framework of Fudenberg and Tirole [11] directly by defining extended mixed strategies over investment thresholds rather than time, even though the underlying demand process is stochastic. ${ }^{17}$

\footnotetext{
${ }^{17}$ A more general dynamic representation allows for firms to choose arbitrary stopping times (see notably Riedel and Steg [29]) which renders the investment game more complex. In the framework we adopt for instance, the investment game remains in the attrition region once it has been initially attained whereas otherwise the demand process exits the attrition regions with positive probability over any time interval.
} 


\section{A.5.1 Strategies, payoffs and equilibrium definitions}

In a dynamic representation of the investment game the continuation payoffs are defined in terms of the current state of the stochastic process $y$, and accordingly denoted $L^{y}(Y), F^{y}(Y)$ and $M^{y}(Y)$, by setting

$$
L^{y}(Y)= \begin{cases}L(Y) & \text { if } \quad Y<y \\ L(y) & \text { if } \quad Y \geq y\end{cases}
$$

and similarly for $F^{y}(Y)$ and $M^{y}(Y)$.

Following Fudenberg and Tirole, a simple strategy for player $i \in\{1,2\}$ in the game starting in state $y$ is a pair of real-valued functions $\left(G_{i}^{y}, \alpha_{i}^{y}\right):[y, \infty) \times[y, \infty) \rightarrow[0,1] \times[0,1]$ such that $(a) G_{i}^{y}$ is non-decreasing and right-continuous, $(b) \alpha_{i}^{y}(Y)>0 \Rightarrow G_{i}^{y}(Y)=1,(c) \alpha_{i}^{y}$ is right-differentiable and $(d)$ if $\alpha_{i}^{y}(Y)=0$ and $Y=\inf \left\{Z \geq Y, \alpha_{i}^{y}(Z)>0\right\}$ then $\alpha_{i}^{y}$ has positive right-derivative at $Y$.

Let $G_{i}^{y-}(Y):=\lim _{Z \rightarrow Y^{-}} G_{i}^{y}(Z)$ denote the left-hand limit of $G_{i}^{y}, a_{i}^{y}(Y)=G_{i}^{y}(Y)-G_{i}^{y-}(Y)$ the magnitude of any jump at $Y$ and let $G_{i}^{y-}(Y)=0, i=1,2$. Let $\mathcal{Y}_{i}(y)=\infty$ if $\alpha_{i}^{y}(Y)=0$ for all $Y \geq y$ and $\mathcal{Y}_{i}(y)=\inf \left\{Z \geq y, \alpha_{i}^{y}(Z)>0\right\}$ otherwise, and let $\mathcal{Y}(y)=\min \left\{\mathcal{Y}_{1}(y), \mathcal{Y}_{2}(y)\right\}$ denote the first threshold at which an investment is certain to occur. Finally let

$$
\mu_{L}(u, v):=\frac{u(1-v)}{u+v-u v} \text { and } \mu_{M}(u, v):=\frac{u v}{u+v-u v} .
$$

Firm payoffs can then be expressed as

$$
\begin{aligned}
& V^{y}\left(\left(G_{i}^{y}, \alpha_{i}^{y}\right),\left(G_{j}^{y}, \alpha_{j}^{y}\right)\right)= \\
& {\left[\int_{y}^{\mathcal{Y}(y)^{-}}\left(L^{y}(s)\left(1-G_{j}^{y}(s)\right) d G_{i}^{y}(s)+F^{y}(s)\left(1-G_{i}^{y}(s)\right) d G_{j}^{y}(s)\right)+\sum_{Z<\mathcal{Y}(y)} a_{i}^{y}(Z) a_{j}^{y}(Z) M^{y}(Z)\right] } \\
& \quad+\left(1-G_{i}^{y-}(\mathcal{Y}(y))\right)\left(1-G_{j}^{y-}(\mathcal{Y}(y))\right) W^{\mathcal{Y}(y)}\left(\left(G_{i}^{y}, \alpha_{i}^{y}\right),\left(G_{j}^{y}, \alpha_{j}^{y}\right)\right),
\end{aligned}
$$

$i, j \in\{1,2\}, i \neq j$ where

$W^{Y}\left(\left(G_{i}^{y}, \alpha_{i}^{y}\right),\left(G_{j}^{y}, \alpha_{j}^{y}\right)\right)=\frac{a_{j}^{y-}(Y)}{1-G_{j}^{y-}(Y)}\left(\left(1-\alpha_{i}^{y}(Y)\right) F^{y}(Y)+\alpha_{i}^{y}(Y) M^{y}(Y)\right)+\frac{1-G_{j}^{y}(Y)}{1-G_{j}^{y-}(Y)} L^{y}(Y)$

if $\mathcal{Y}_{i}(y)<\mathcal{Y}_{j}(y)$,

$$
=\frac{a_{i}^{y-}(Y)}{1-G_{i}^{y-}(Y)}\left(\left(1-\alpha_{j}^{y}(Y)\right) L^{y}(Y)+\alpha_{j}^{y}(Y) M^{y}(Y)\right)+\frac{1-G_{i}^{y}(Y)}{1-G_{i}^{y-}(Y)} F^{y}(Y)
$$


if $\mathcal{Y}_{i}(y)>\mathcal{Y}_{j}(y)$ and

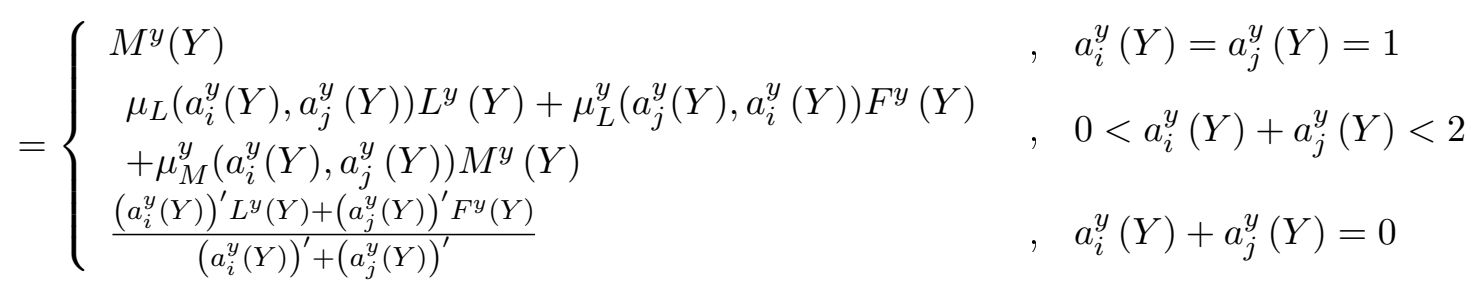

if $\mathcal{Y}_{i}(y)=\mathcal{Y}_{j}(y)$

For a given $y$ a pair of simple strategies $\left(\left(G_{1}^{y}, \alpha_{1}^{y}\right),\left(G_{2}^{y}, \alpha_{2}^{y}\right)\right)$ is a Nash equilibrium of the game $\left(\{1,2\},\left(G_{1}^{y}, \alpha_{1}^{y}\right) \times\left(G_{2}^{y}, \alpha_{2}^{y}\right),\left(V^{y}, V^{y}\right)\right)$ if $\left(G_{i}^{y}, \alpha_{i}^{y}\right)$ maximizes $V^{y}\left(\cdot,\left(G_{i}^{y}, \alpha_{i}^{y}\right)\right), i, j \in\{1,2\}$, $i \neq j$. A collection of simple strategies $\left(\left(G_{i}^{y}(Y), \alpha_{i}^{y}(Y)\right)\right)_{y>0}$ is said to be consistent if for $y \leq$ $Y \leq Z, G_{i}^{y}(Z)=G_{i}^{y}(Y)+\left(1-G_{i}^{y}(Y)\right) G_{i}^{Y}(Z)$ and $\alpha_{i}^{y}(Z)=\alpha_{i}^{Y}(Z)$. The consistent strategies $\left(\left(G_{1}^{y}(Y), \alpha_{1}^{y}(Y)\right)\right)_{y>0}$ and $\left(\left(G_{2}^{y}(Y), \alpha_{2}^{y}(Y)\right)\right)_{y>0}$ are a perfect equilibrium if the simple strategies $\left(G_{1}^{y}(Y), \alpha_{1}^{y}(Y)\right)$ and $\left(G_{2}^{y}(Y), \alpha_{2}^{y}(Y)\right)$ are a Nash equilibrium for every $y$.

\section{A.5.2 Equilibrium}

$K<\widehat{K}$ case In the attrition range firms do not profit from using mixed strategies extensions $\left(\alpha_{i}^{y}>0\right)$ to coordinate simultaneous investments in a waiting game. Equilibrium strategies are therefore essentially derived from the unconditional strategies $G_{a}(Y)$ and $G_{b}(Y)$ depending upon whether $K \leq \widehat{K}$ or $\widetilde{K}<K<\widehat{K}$. Therefore, letting $G_{a}^{y}(Y):=\frac{G_{a}(Y)-G_{a}(y)}{1-G_{a}(y)}$ and $G_{b}^{y}(Y):=$ $\frac{G_{b}(Y)-G_{b}(y)}{1-G_{b}(y)},\left(G_{i}^{y}(Y), \alpha_{i}^{y}(Y)\right)=\left(G_{a}^{y}(Y), 0\right)$ and $\left(G_{i}^{y}(Y), \alpha_{i}^{y}(Y)\right)=\left(G_{b}^{y}(Y), 0\right)$ are equilibrium strategies in these two subcases respectively.

$K>\widehat{K}$ case In the preemption range there are two subcases that we consider successively.

$\widehat{K}<K<I$ subcase This is the case represented in Figure 3 whose key features are that the preemption range (over which $\left.L^{y}(Y)>F^{y}(Y)\right)$ is the bounded open interval $\left(Y_{P}, Y_{P^{\prime}}\right) \subset\left(Y_{P}, Y_{F}\right)$, and that if a threshold beyond this range is reached (off the equilibrium path), firms play a waiting game as $F^{y}(Y)>L^{y}(Y)$ for $Y>Y_{P^{\prime}}$. In a dynamic representation of the game equilibrium strategies must account explicitly for this possibility.

At any $y>Y_{P^{\prime}}$ the payoff to leading lies below the follower payoff, but it is not monotonic. In $\left(Y_{P^{\prime}}, Y_{F}\right)$ there exists a unique threshold $Y_{S^{\prime}}$ such that $L^{y}\left(Y_{S^{\prime}}\right)=L^{y}\left(Y_{S}\right)$. The leader payoff is decreasing only over $\left(Y_{P^{\prime}}, Y_{S^{\prime}}\right) \cup\left(Y_{S}, \infty\right)$, and it is this range that constitutes the support of mixed 
strategies. The attrition subgame is then solved similarly to the $\widehat{K}<K<\widetilde{K}$ case in Section A.3 yielding unconditional distributions

$$
G_{c}(Y)=1-\exp \int_{Y_{P^{\prime}}}^{Y} \frac{\left[L^{y}(s)\right]^{\prime}}{F\left(\max \left\{Y, Y_{F}\right\}\right)-L^{y}(s)} d s
$$

and

$$
G_{d}(Y)= \begin{cases}0 & \text { if } Y<Y_{p^{\prime}} \\ G_{c}(Y) & \text { if } Y_{P^{\prime}} \leq Y \leq Y_{S^{\prime}} \\ G_{c}\left(Y_{S^{\prime}}\right) & \text { if } Y_{S^{\prime}}<Y<Y_{S} \\ G_{c}\left(Y_{S^{\prime}}\right)+\left(1-G_{c}\left(Y_{S^{\prime}}\right)\right) G_{a}(Y) & \text { if } Y \geq Y_{S}\end{cases}
$$

so that the conditional distribution is $G_{d}^{y}(Y):=\frac{G_{d}(Y)-G_{d}(y)}{1-G_{d}(y)}$.

If $y$ lies in the preemption range the reasoning is standard and results in firms investing immediately and using the strategy extensions to coordinate simultaneous investment.

To summarize equilibrium strategies in this case are $\left(G_{i}^{y}(Y), \alpha_{i}^{y}(Y)\right)$ with

$$
\begin{aligned}
& G_{i}^{y}(Y)=\left\{\begin{array}{ccc}
0 & \text { if } & Y<Y_{P} \\
1 & \text { if } & Y_{P} \leq Y<Y_{P^{\prime}} \\
G_{d}^{y}(Y) & \text { if } & Y \geq Y_{P^{\prime}}
\end{array}\right. \\
& \alpha_{i}^{y}(Y)=\left\{\begin{array}{ccc}
0 & \text { if } & Y<Y_{P} \\
\frac{L^{y}(Y)-F^{y}(Y)}{L^{y}(Y)-M^{y}(Y)} & \text { if } & Y_{P} \leq Y<Y_{P^{\prime}} \\
0 & \text { if } & Y \geq Y_{P^{\prime}}
\end{array}\right.
\end{aligned}
$$

for $i \in\{1,2\}$.

$K \geq I$ subcase Here $L^{y}(Y)>F^{y}(Y)$ over $\left(Y_{P}, Y_{F}\right)$ so the investment game is a standard case of preemption over this range. A specificity of the investment game studied here is that for $K>I, M^{y}$ lies strictly above $F^{y}$ over $\left[Y_{F}, \infty\right)$. Equilibrium strategies are thus those of a standard real option game, yielding $\left(G_{i}^{y}(Y), \alpha_{i}^{y}(Y)\right)$ with

$$
\begin{aligned}
& G_{i}^{y}(Y)=\left\{\begin{array}{ccc}
0 & \text { if } \quad Y<Y_{P} \\
1 & \text { if } \quad Y \geq Y_{P}
\end{array},\right. \\
& \alpha_{i}^{y}(Y)=\left\{\begin{array}{ccc}
0 & \text { if } & Y<Y_{P} \\
\frac{L^{y}(Y)-F^{y}(Y)}{L^{y}(Y)-M^{y}(Y)} & \text { if } & Y_{P} \leq Y<Y_{F} \\
1 & \text { if } & Y \geq Y_{F}
\end{array}\right.
\end{aligned}
$$

for $i \in\{1,2\}$. 


\section{A.6 Imitation cost, consumer surplus, and welfare (Proposition 3)}

The proof of Proposition 3 involves several steps. First, to establish (iii) by determining the optimal imitation cost level $K_{P}$ in the closure of the preemption regime (for $K \geq \widehat{K}$ ). Next we prove part $(i i)$ by showing that $W(K)$ is increasing over $[0, \widetilde{K})$. Finally for part $(i)$ we establish the existence of a local optimum of welfare under attrition $\left(\widetilde{K} \leq K_{A}<\widehat{K}\right)$ and compare optimal welfare in the attrition and preemption regimes.

Optimal imitation cost $K_{P}$

Suppose that $K \geq \widehat{K}$ so investment thresholds are $Y_{P}$ and $Y_{F}$. The social welfare function (8) then has the form

$$
W(K)=\left(\frac{\pi_{M}+\mathrm{CS}_{M}}{r-\alpha} Y_{P}-I\right)\left(\frac{y}{Y_{P}}\right)^{\beta}+\left(\frac{\left(2 \pi_{D}+\mathrm{CS}_{D}\right)-\left(\pi_{M}+\mathrm{CS}_{M}\right)}{r-\alpha} Y_{F}-K\right)\left(\frac{y}{Y_{F}}\right)^{\beta} .
$$

Noting that $Y_{P}$ and $Y_{F}$ are functions of $K$ with $Y_{P} \leq Y_{L}$ and $\lim _{K \rightarrow \widehat{K}} Y_{P}=Y_{L}$. Using the preemption equilibrium condition $L\left(Y_{P}\right)=F\left(Y_{F}\right)$ and implicit differentiation to obtain a useful expression for the ratio $\left(Y_{F} / Y_{P}\right)^{\beta}$, after several steps of calculations (see Section A.9), for $K>\widehat{K}$ the derivative of welfare can be expressed

$$
W^{\prime}(K)=\left(\frac{\mathrm{CS}_{M}}{\pi_{M}}\left(\beta \frac{\pi_{M}}{\pi_{D}}+\left(\beta \frac{\pi_{M}}{\pi_{D}}-(\beta-1)\right) \frac{Y_{L}}{Y_{L}-Y_{P}}\right)-\beta \frac{\mathrm{CS}_{D}}{\pi_{D}}-2\right)\left(\frac{y}{Y_{F}}\right)^{\beta} .
$$

If $\mathrm{CS}_{M}=0$, the $Y_{L}$ and $Y_{P}$ terms vanish and it is straightforward to see that $W^{\prime}(K)<0$ so that $\widehat{K}$ is a maximum in this case. Otherwise $\mathrm{CS}_{M}>0$ and the behavior of $W(K)$ for $K>\widehat{K}$ can be characterized as follows. Since $\lim _{K \rightarrow \widehat{K}^{+}} Y_{P}=Y_{L}$, we have $\lim _{K \rightarrow \widehat{K}} W^{\prime}(K)=+\infty$. Moreover both $\left(Y_{L} /\left(Y_{L}-Y_{P}\right)\right)$ and $\left(y / Y_{F}\right)$ are decreasing in $K$ so $W$ is concave over this range. So long as $\lim _{K \rightarrow \infty} W^{\prime}(K)<0$ therefore, there exists a unique root $K_{P}>\widehat{K}$ that constitutes an interior optimum, and as $\lim _{K \rightarrow \infty} Y_{P}=(r-\alpha) I / \pi_{M}=((\beta-1) / \beta) Y_{L}$, this occurs if

$$
\left(\beta^{2} \frac{\pi_{M}}{\pi_{D}}-(\beta-1)^{2}\right) \frac{\mathrm{CS}_{M}}{\pi_{M}}-\beta \frac{\mathrm{CS}_{D}}{\pi_{D}}-2<0 .
$$

If (19) does not hold, set $K_{P}=\infty$. Taken as a function of $\beta$ the left-hand side of (19) is a quadratic $\Delta(\beta)$, with $\Delta(1)=\left(\mathrm{CS}_{M}-\mathrm{CS}_{D}-2 \pi_{D}\right) / \pi_{D}<0$ and $\lim _{\infty} \Delta(\beta)=\infty$. Therefore there exists a unique $\beta_{0}>1$ such that $\Delta\left(\beta_{0}\right)=0$.

To summarize, we have so far characterized solutions to the constrained optimization problem $\max _{K \geq \widehat{K}} W(K)$ and shown that there is a unique optimum $K_{P}$ in the extended real line and there exists a unique $\beta_{0}>1$ such that $K_{P}$ is finite if and only if $\beta<\beta_{0}$. 
Next, several steps of calculation (again see Section A.9 for details) establish that if the imitation cost is set at $K_{P}$, the corresponding (optimum) preemption threshold has the form $Y_{P}^{*}=\psi Y_{L}$ where

$$
\psi=\left\{\begin{array}{l}
\frac{\frac{\mathrm{CS}_{D}-\mathrm{CS}_{M}}{\pi_{D}}+\frac{2}{\beta}}{\frac{\mathrm{CS}_{D}}{\pi_{D}}-\frac{\beta-1}{\beta} \frac{\mathrm{CS}_{M}}{\pi_{M}}+\frac{2}{\beta}}, \beta<\beta_{0} \\
\frac{\beta}{\beta-1}, \beta \geq \beta_{0}
\end{array}\right.
$$

We have $\psi \in\left[\frac{\beta-1}{\beta}, 1\right]$ with $\psi=1$ if $\operatorname{CS}_{M}=0$ and $\psi=(\beta-1) / \beta$ if $\beta \geq \beta_{0}$, establishing part (iii).

Note that the optimal preemption threshold $Y_{P}^{*}$ satisfies $Y_{P}^{*} \in\left[Y_{\mathrm{NPV}}, Y_{L}\right]$ where $Y_{\mathrm{NPV}}:=$ $(r-\alpha) I / \pi_{M}$ is the myopic Marshallian investment trigger. The optimal level of welfare under preemption can be shown to be

$$
W\left(K_{P}\right)=\frac{\mathrm{CS}_{M}}{\pi_{M}} \frac{\psi^{1-\beta}}{1-\psi} \frac{I}{\beta-1}\left(\frac{Y_{0}}{Y_{L}}\right)^{\beta}
$$

and this latter expression is useful later in the proof of Proposition 4.

\section{Lower bound on socially optimal imitation cost}

If $K<\widetilde{K}$, firms randomize investment triggers over $\left[Y_{S}, \infty\right)$ according to the distribution $G_{a}(Y)$ and imitator entry is immediate. Observe first that by Proposition 2 industry value is lower at any $K$ than at $\widehat{K}$, so it is sufficient to show that the expected consumer surplus for $K<\widetilde{K}$ is bounded above by its value at $\widehat{K}$ as well. The lower bound of the entry threshold distribution under attrition in this case is $Y_{S}=(\beta(r-\alpha) I) /\left((\beta-1) \pi_{D}\right)$, whereas at $\widehat{K}$ innovator and imitator entry both occur earlier, at the thresholds $Y_{L}$ and $\widehat{Y}_{F}:=(\beta(r-\alpha) \widehat{K}) /\left((\beta-1) \pi_{D}\right)$. Therefore, both investments occur later under this type of attrition regime resulting in lower consumer surplus and $K<\widetilde{K}$ cannot be a social optimum.

\section{Existence of local optimum under attrition and comparison}

Finally consider the behavior of $W(K)$ just to the left of $\widehat{K}$. Since $\mathbb{E} V\left(\widetilde{Y}_{1}, \widetilde{Y}_{2}\right)$ is maximized at $\widehat{K}$, the value of $\lim _{K \rightarrow \widehat{K}^{-}} W^{\prime}(K)$ depends only on the left derivatives of the consumer surplus terms at $\widehat{K}$. Consider first the derivative of the third term, consumer surplus from imitation. Developing it yields

$$
\frac{\mathrm{CS}_{D}-\mathrm{CS}_{M}}{r-\alpha}\left[Y_{F}\right]^{-(\beta-1)} y^{\beta}(\underbrace{G_{\wedge}\left(Y_{S^{\prime}} ; K\right)}_{\text {lagged imitator entry }}+\underbrace{\int_{Y_{S}}^{\infty}\left(Y_{F} / s\right)^{\beta-1} d G_{\wedge}(s ; K)}_{\text {immediate imitator entry }}) .
$$


To determine the value of the left derivative at $\widehat{K}$ of this expression recall that the distribution of entry thresholds is by $G_{\wedge}(Y ; K)=1-\left(1-G_{b}(Y)\right)^{2}$. Since $G_{b}\left(Y_{S^{\prime}} ; \widehat{K}\right)=1, G_{\wedge}\left(Y_{S^{\prime}} ; \widehat{K}\right)=1$ and moreover $\partial G_{\wedge} / \partial Y=2\left(1-G_{b}\right)\left(\partial G_{b} / \partial Y\right), \partial G_{\wedge}\left(Y_{S^{\prime}} ; \widehat{K}\right) / \partial K \mid=0$. Only the direct effect of $K$ on $Y_{F}$ therefore remains at $\widehat{K}$, and Similar reasoning holds for the consumer surplus from innovation term in (8) (except that the is no direct effect since $Y_{L}$ is independent of $K$ ). Therefore,

$$
\lim _{K \rightarrow \widehat{K}_{-}} W^{\prime}(K)=-\beta \frac{\mathrm{CS}_{D}-\mathrm{CS}_{M}}{\pi_{D}} Y_{F}^{-\beta} y^{\beta} \frac{\partial Y_{F}}{\partial K} \leq 0 .
$$

As $W$ is continuous we conclude that if $\mathrm{CS}_{D}>\mathrm{CS}_{M}$ there exists a local optimum imitation cost level $K_{A}$ in $(\widetilde{K}, \widehat{K})$.

As $\lim _{K \rightarrow \widehat{K}_{-}} d W(K) / d K<0$ for $\mathrm{CS}_{D}>\mathrm{CS}_{M}$ and $\lim _{K \rightarrow \widehat{K}+} d W(K) / d K>0$ for $\mathrm{CS}_{M}>0$ we conclude that for $\left(\mathrm{CS}_{D}-\mathrm{CS}_{M}\right) \mathrm{CS}_{M}>0$ welfare has local maxima in both the (upper) attrition and preemption ranges. Either type of local maximum can be a global maximum depending on the relative magnitude of the consumer surplus resulting from innovation and imitation, which establishes part $(i)$.

\section{A.7 Sufficient condition for preemption to be optimal (Proposition 4)}

The result is established by first deriving an upper bound for the level of welfare realized in the attrition regime, which is then compared with the lower bound of the welfare obtained under preemption so as to obtain a tractable sufficient condition.

Upper bound for welfare under attrition

Under attrition, innovation and imitation occur at thresholds $\widetilde{Y}_{A}$ and $\widetilde{Y}_{F}=\min \left\{\widetilde{Y}_{A}, Y_{F}\right\}$ so the expected social welfare $(8)$ is

$$
W(K)=\mathbb{E}\left(\frac{\mathrm{CS}_{M}+\pi_{M}}{r-\alpha} \widetilde{Y}_{A}-I\right)\left(\frac{y}{\widetilde{Y}_{A}}\right)^{\beta}+\mathbb{E}\left(\frac{\left(\mathrm{CS}_{D}+2 \pi_{D}\right)-\left(\mathrm{CS}_{M}+\pi_{M}\right)}{r-\alpha} \widetilde{Y}_{F}-K\right)\left(\frac{y}{\widetilde{Y}_{F}}\right)^{\beta} .
$$

The term in the first summand is quasiconcave in the investment threshold with a maximum at $(\beta(r-\alpha) I) /\left((\beta-1)\left(\mathrm{CS}_{M}+\pi_{M}\right)\right) \leq Y_{L}$ whereas given the support of the attrition distribution $\widetilde{Y}_{A} \geq Y_{L}$. Therefore

$$
\begin{aligned}
\mathbb{E}\left(\frac{\mathrm{CS}_{M}+\pi_{M}}{r-\alpha} \widetilde{Y}_{A}-I\right)\left(\frac{y}{\widetilde{Y}_{A}}\right)^{\beta} & \leq\left(\frac{\mathrm{CS}_{M}+\pi_{M}}{r-\alpha} Y_{L}-I\right)\left(\frac{y}{Y_{L}}\right)^{\beta} \\
& \leq\left(\beta \frac{\mathrm{CS}_{M}}{\pi_{M}}+1\right) \frac{I}{\beta-1}\left(\frac{y}{Y_{L}}\right)^{\beta}
\end{aligned}
$$


The second summand can be bounded using the assumption that the static entry incentive is excessive,

$$
\mathbb{E}\left(\frac{\left(\mathrm{CS}_{D}+2 \pi_{D}\right)-\left(\mathrm{CS}_{M}+\pi_{M}\right)}{r-\alpha} \widetilde{Y}_{F}-K\right)\left(\frac{y}{\widetilde{Y}_{F}}\right)^{\beta} \leq \mathbb{E}\left(\frac{\pi_{D}}{r-\alpha} \widetilde{Y}_{F}-K\right)\left(\frac{y}{\widetilde{Y}_{F}}\right)^{\beta}
$$

The term on the right-hand side is the expected follower payoff in equilibrium, $\mathbb{E} F\left(\widetilde{Y}_{F}\right)$. Moreover in equilibrium payoffs are constant over the support of mixed strategies so $\mathbb{E} F\left(\widetilde{Y}_{F}\right)=$ $\max \left\{L\left(Y_{L}\right), L\left(Y_{S}\right)\right\}$, and this latter term is maximized for $K=\widehat{K}$ by Proposition 2. Therefore

$$
\mathbb{E}\left(\frac{\left(\mathrm{CS}_{D}+2 \pi_{D}\right)-\left(\mathrm{CS}_{M}+\pi_{M}\right)}{r-\alpha} \widetilde{Y}_{F}-K\right)\left(\frac{y}{\widetilde{Y}_{F}}\right)^{\beta} \leq \frac{\widehat{K}}{\beta-1}\left(\frac{y}{\widehat{Y}_{F}}\right)^{\beta}
$$

where $\left.\widehat{Y}_{F}:=(\beta(r-\alpha) \widehat{K}) /\left((\beta-1) \pi_{D}\right)\right)=(\widehat{K} / I)\left(\pi_{M} / \pi_{D}\right) Y_{L}$. Using (12) to substitute for $(\widehat{K} / I)^{-(\beta-1)}$ gives

$$
\mathbb{E}\left(\frac{\left(\mathrm{CS}_{D}+2 \pi_{D}\right)-\left(\mathrm{CS}_{M}+\pi_{M}\right)}{r-\alpha} \tilde{Y}_{F}-K\right)\left(\frac{y}{\widetilde{Y}_{F}}\right)^{\beta} \leq \frac{1}{1+\beta\left(\frac{\pi_{M}}{\pi_{D}}-1\right)} \frac{I}{\beta-1}\left(\frac{y}{Y_{L}}\right)^{\beta} .
$$

Combining (20) and (21) yields

$$
W(K) \leq\left(\beta \frac{\mathrm{CS}_{M}}{\pi_{M}}+1+\frac{1}{1+\beta\left(\frac{\pi_{M}}{\pi_{D}}-1\right)}\right) \frac{I}{\beta-1}\left(\frac{y}{Y_{L}}\right)^{\beta}
$$

for $K \leq \widehat{K}$

Comparison of attrition and preemption welfare

The optimal value of expected welfare under preemption is

$$
W\left(K_{P}\right)=\frac{\mathrm{CS}_{M}}{\pi_{M}} \frac{1}{\psi^{\beta-1}-\psi^{\beta}} \frac{I}{\beta-1}\left(\frac{y}{Y_{L}}\right)^{\beta}
$$

(see Section A.9). Preemption is therefore socially optimal if

$$
\frac{\mathrm{CS}_{M}}{\pi_{M}}\left(\frac{1}{\psi^{\beta-1}-\psi^{\beta}}-\beta\right) \geq 1+\frac{1}{1+\beta\left(\frac{\pi_{M}}{\pi_{D}}-1\right)} .
$$

This condition is difficult to interpret however since $\psi$ is itself a function of parameters including $\mathrm{CS}_{D}$ and $\pi_{D}$. We therefore derive a conservative (it is only tight in the limit as $\beta \rightarrow 1$ ) but more tractable bound. 
Taken as a function of $\psi$ over $(0,1), 1 /\left(\psi^{\beta-1}-\psi^{\beta}\right)$ is a strictly convex function that is minimized at $\psi_{0}:=(\beta-1) / \beta$. Substituting this value into the left-hand side of $(22)$ and using $1 /\left(1+\beta\left(\left(\pi_{M} / \pi_{D}\right)-1\right)\right) \leq 1$ yields after rearranging the stronger sufficient condition

$$
\frac{\mathrm{CS}_{M}}{\pi_{M}} \geq \frac{2}{\beta} \frac{1}{\left(\frac{\beta}{\beta-1}\right)^{\beta-1}-1}=: \Omega(\beta) .
$$

The right-hand side of (23) can be characterized, first by observing that by l'Hôpital's rule, $\lim _{\beta \rightarrow 1}\left(\frac{\beta}{\beta-1}\right)^{\beta-1}=1$ and $\lim _{\beta \rightarrow \infty}\left(\frac{\beta}{\beta-1}\right)^{\beta-1}=$ e so $\lim _{\beta \rightarrow 1} \Omega(1)=\infty$ and $\lim _{\beta \rightarrow \infty} \Omega(\beta)=0$.

Moreover, $2 / \beta$ is decreasing and $d\left[(\beta /(\beta-1))^{\beta-1}\right] / d \beta=\left[(\beta /(\beta-1))^{\beta-1}\right](-(1 / \beta)+\ln (\beta /(\beta-1)))$ which is positive since $\ln (\beta /(\beta-1))>1 / \beta$ by the logarithm inequality. Therefore $\Omega^{\prime}(\beta)<0$.

\section{A.8 Buyout and licensing}

\section{Buyouts and attrition}

To verify the claim that attrition does not occur if $\pi_{M} / \pi_{D} \geq \beta+1$ suppose that $K=0$ (the imitation cost most favorable for attrition). The leader payoff $L_{\mathrm{B}}(Y)$ is maximized at the threshold $Y_{B}=(\beta(r-\alpha) I) /\left((\beta-1)\left(\pi_{M}-\pi_{D}\right)\right)$. The investment game is (weakly) preemptive if $L_{\mathrm{B}}\left(Y_{B}\right) \geq F\left(Y_{B}\right)$, that is if

$$
\left(\frac{\pi_{M}-\pi_{D}}{r-\alpha} Y_{B}-I\right)\left(\frac{y}{Y_{B}}\right)^{\beta} \geq \frac{\pi_{D}}{r-\alpha} Y_{B}\left(\frac{y}{Y_{B}}\right)^{\beta}
$$

which yields the desired condition.

\section{Proposition 6}

We first verify that a buyout is the preferred instrument. The condition $L_{\mathrm{B}}(Y) \geq L_{\mathrm{L}}(Y)$ works out to

$$
\left(\frac{\pi_{M}-\pi_{D}}{r-\alpha} \max \left\{Y, Y_{F}\right\}-K_{I}\right)\left(\frac{y}{\max \left\{Y, Y_{F}\right\}}\right)^{\beta} \geq\left(\frac{\pi_{D}}{r-\alpha} \max \left\{Y, Y_{F}\right\}-K_{0}-K_{I}\right)\left(\frac{y}{\max \left\{Y, Y_{F}\right\}}\right)^{\beta}
$$

which holds because of the efficiency effect $\pi_{M}>2 \pi_{D}$.

That buyouts increase firm profit for $K<\widehat{K}$ follows from $L_{\mathrm{B}}(Y)>L(Y)$ and the rent dissipation property of attrition and preemption.

Similarly, licensing (provided $K_{I}>0$ ) increases firm profit while leaving the timing of imitation unchanged. It therefore remains to verify that licensing results in earlier innovation. Let $\widehat{K}_{L}<\widehat{K}$ 
denote the critical threshold that separates attrition and preemption in the presence of licensing, which solves $L_{\mathrm{L}}\left(Y_{L}\right)=F\left(Y_{F}\right)$ (as licensing only has a level effect on the leader payoff for $Y<Y_{F}$ the payoff $L_{\mathrm{L}}$ is maximized at $\left.Y_{L}\right)$. For $K \geq \widehat{K}_{L}$, as $L_{\mathrm{L}}(Y)>L(Y)$ allowing licensing results in innovation at a threshold that is either lower than the preemption threshold without licensing or weakly lower than the previous possible innovation thresholds. Otherwise if $K<\widehat{K}_{L}$, the industry is in an attrition regime both with and without licensing and the distribution of innovation thresholds shifts left with licensing.

\section{A.9 Additional derivations}

This section details some of the lengthier derivations necessary to determine $K_{P}$ and $Y_{P}$ (the optimal imitation cost and preemption threshold within the extended preemption range) and $W\left(K_{P}\right)$ (the optimal welfare under extended preemption).

Characterization of $Y_{P}$ and $d Y_{P} / d K$

The condition $L\left(Y_{P}\right)=F\left(Y_{F}\right)$ implicitly defines the preemption threshold $Y_{P}$ as a $\mathcal{C}^{1}$ function

of $K$ over $(\widehat{K}, \infty)$ (see Section A.2). Dividing by $y^{\beta}$ and grouping $Y_{F}$ yields the more compact form of this condition,

$$
\begin{aligned}
\left(\frac{\pi_{M}}{r-\alpha} Y_{P}-I\right) Y_{P}^{-\beta} & =\left(\frac{\pi_{M}}{r-\alpha} Y_{F}-K\right) Y_{F}^{-\beta} \\
& =\left(\frac{\beta}{\beta-1} \frac{\pi_{M}}{\pi_{D}}-1\right)\left(\frac{\beta-1}{\beta} \frac{\pi_{D}}{r-\alpha}\right)^{\beta} K^{-(\beta-1)}
\end{aligned}
$$

This condition has the form $f\left(Y_{P}\right)=g(K)$, so that $d Y_{P} / d K=g^{\prime}(K) / f^{\prime}\left(Y_{P}\right)$ where

$$
f^{\prime}\left(Y_{P}\right)=\left(-(\beta-1) \frac{\pi_{M}}{r-\alpha} Y_{P}+\beta I\right) Y_{P}^{-\beta-1}
$$

and

$$
g^{\prime}(K)=-\frac{\beta-1}{K} g(K)<0
$$

Any preemption threshold $Y_{P}$ satisfies $Y_{P}<Y_{L}$, so $f^{\prime}\left(Y_{P}\right)>0$. Finally, using the identity $g(K)=f\left(Y_{P}\right)$ and developing yields

$$
\frac{d Y_{P}}{d K}=-\frac{Y_{P}-Y_{\mathrm{NPV}}}{Y_{L}-Y_{P}}\left(\frac{Y_{P}}{K}\right) .
$$

Interior preemption optimum $K_{P}$ 
Suppose that condition (19) holds so that the preemption optimum is known to be an interior solution. To derive its explicit form, first substitute $Y_{F}$ in the social welfare function (18) to get

$$
\begin{aligned}
W(K)=\left(\frac{\mathrm{CS}_{M}+}{r-\alpha} \pi_{M}\right. & \left.Y_{P}-I\right)\left(\frac{y}{Y_{P}}\right)^{\beta} \\
& \quad+\left(\frac{\beta}{\beta-1} \frac{\left(\mathrm{CS}_{D}+2 \pi_{D}\right)-\left(\mathrm{CS}_{M}+\pi_{M}\right)}{\pi_{D}}-1\right)\left(\frac{\beta-1}{\beta} \frac{\pi_{D}}{r-\alpha}\right)^{\beta} y^{\beta} K^{-(\beta-1)} .
\end{aligned}
$$

Consider then the problem $\max _{K \geq \widehat{K}} W(K)$. We have

$$
\begin{aligned}
& W^{\prime}(K)=(-(\beta-1)\left.\frac{\mathrm{CS}_{M}+\pi_{M}}{r-\alpha} Y_{P}+\beta I\right)\left(\frac{y}{Y_{P}}\right)^{\beta} \frac{1}{Y_{P}} \frac{d Y_{P}}{d K} \\
&-\left(\beta \frac{\left(\mathrm{CS}_{D}+2 \pi_{D}\right)-\left(\mathrm{CS}_{M}+\pi_{M}\right)}{\pi_{D}}-(\beta-1)\right)\left(\frac{\beta}{\beta-1} \frac{r-\alpha}{\pi_{D}}\right)^{-\beta} y^{\beta} K^{-\beta} .
\end{aligned}
$$

At an interior optimum the socially optimal imitation cost $K_{P}$ satisfies the condition $W_{P}^{\prime}\left(K_{P}\right)=0$ but it is convenient to obtain an expression for the socially optimal preemption threshold $Y_{P}^{*}$ instead.

Substituting for $d Y_{P} / d K$ and normalizing the first-order condition yields

$$
\begin{aligned}
& \left((\beta-1) \frac{\mathrm{CS}_{M}+\pi_{M}}{r-\alpha} Y_{P}^{*}-\beta I\right) \frac{Y_{P}^{*}-Y_{\mathrm{NPV}}}{Y_{L}-Y_{P}^{*}}\left[Y_{P}^{*}\right]^{-\beta} \\
& \quad-\left(\beta \frac{\left(\mathrm{CS}_{D}+2 \pi_{D}\right)-\left(\mathrm{CS}_{M}+\pi_{M}\right)}{\pi_{D}}-(\beta-1)\right)\left(\frac{\beta}{\beta-1} \frac{r-\alpha}{\pi_{D}}\right)^{-\beta} K_{P}^{-(\beta-1)}=0
\end{aligned}
$$

and substituting the preemption condition (24) gives a condition in $Y_{P}^{*}$ only,

$$
\begin{aligned}
\left((\beta-1) \frac{\mathrm{CS}_{M}+\pi_{M}}{r-\alpha} Y_{P}^{*}-\beta I\right) & \frac{Y_{P}^{*}-Y_{\mathrm{NPV}}}{Y_{L}-Y_{P}^{*}} \\
& -\left(\beta \frac{\left(\mathrm{CS}_{D}+2 \pi_{D}\right)-\left(\mathrm{CS}_{M}+\pi_{M}\right)}{\pi_{D}}-(\beta-1)\right) \frac{\frac{\pi_{M}}{r-\alpha} Y_{P}^{*}-I}{\frac{\beta}{\beta-1} \frac{\pi_{M}}{\pi_{D}}-1}=0 .
\end{aligned}
$$

Noting that $Y_{P}^{*}-Y_{\mathrm{NPV}}=\frac{r-\alpha}{\pi_{M}}\left(\frac{\pi_{M}}{r-\alpha} Y_{P}^{*}-I\right)$ and rearranging then gives

$$
\begin{aligned}
\left(\frac{\mathrm{CS}_{M}+\pi_{M}}{\pi_{M}} Y_{P}^{*}-Y_{L}\right)\left(\frac{\beta}{\beta-1} \frac{\pi_{M}}{\pi_{D}}\right. & -1) \\
& =\left(Y_{L}-Y_{P}^{*}\right)\left(\frac{\beta}{\beta-1} \frac{\left(\mathrm{CS}_{D}+2 \pi_{D}\right)-\left(\mathrm{CS}_{M}+\pi_{M}\right)}{\pi_{D}}-1\right) .
\end{aligned}
$$

There is a unique solution to this condition which can be expressed as $Y_{P}^{*}=\psi Y_{L}$ where 


$$
\psi=\frac{\frac{\mathrm{CS}_{D}-\mathrm{CS}_{M}}{\pi_{D}}+\frac{2}{\beta}}{\frac{\mathrm{CS}_{D}}{\pi_{D}}-\frac{\beta-1}{\beta} \frac{\mathrm{CS}_{M}}{\pi_{M}}+\frac{2}{\beta}} .
$$

Note that the condition $Y_{P}^{*}>Y_{\mathrm{NPV}}$ is equivalent to $\psi>(\beta-1) / \beta$ which yields condition (19) in the text.

Value of optimal welfare under preemption $W\left(K_{P}\right)$

The optimal social welfare can now be evaluated as follows. Using (24) to substitute in the expression for $W\left(K_{P}\right)$ above yields

$$
\begin{aligned}
& W_{P}\left(K_{P}\right)= \\
& \frac{\left(\frac{\mathrm{CS}_{M}+\pi_{M}}{r-\alpha} Y_{P}^{*}-I\right)\left(\frac{\beta}{\beta-1} \frac{\pi_{M}}{\pi_{D}}-1\right)+\left(\frac{\beta}{\beta-1} \frac{\left(\mathrm{CS}_{D}+2 \pi_{D}\right)-\left(\mathrm{CS}_{M}+\pi_{M}\right)}{\pi_{D}}-1\right)\left(\frac{\pi_{M}}{r-\alpha} Y_{P}^{*}-I\right)}{\frac{\beta}{\beta-1} \frac{\pi_{M}}{\pi_{D}}-1}\left(\frac{y}{Y_{P}^{*}}\right)^{\beta} .
\end{aligned}
$$

Substituting for $Y_{P}^{*}=(\beta(r-\alpha) \psi I) /\left((\beta-1) \pi_{M}\right)\left(=\psi Y_{L}\right)$ and factoring $I$,

$$
\begin{aligned}
& W_{P}\left(K_{P}\right)= \\
& \frac{\left(\frac{\beta}{\beta-1} \frac{\mathrm{CS}_{M}+\pi_{M}}{\pi_{M}} \psi-1\right)\left(\frac{\beta}{\beta-1} \frac{\pi_{M}}{\pi_{D}}-1\right)+\left(\frac{\beta}{\beta-1} \frac{\left(\mathrm{CS}_{D}+2 \pi_{D}\right)-\left(\mathrm{CS}_{M}+\pi_{M}\right)}{\pi_{D}}-1\right)\left(\frac{\beta}{\beta-1} \psi-1\right)}{\frac{\beta}{\beta-1} \frac{\pi_{M}}{\pi_{D}}-1} \psi^{-\beta} I\left(\frac{y}{Y_{L}}\right)^{\beta} .
\end{aligned}
$$

Then after substituting the expression for $\psi$ and some algebra,

$$
W_{P}\left(K_{P}\right)=\frac{\mathrm{CS}_{M}}{\pi_{M}} \frac{\psi^{-(\beta-1)}}{1-\psi} \frac{I}{\beta-1}\left(\frac{y}{Y_{L}}\right)^{\beta} .
$$

